\title{
WEAK BIMONOIDS IN DUOIDAL CATEGORIES
}

\author{
YUANYUAN CHEN AND GABRIELLA BÖHM
}

\begin{abstract}
Weak bimonoids in duoidal categories are introduced. They provide a common generalization of bimonoids in duoidal categories and of weak bimonoids in braided monoidal categories. Under the assumption that idempotent morphisms in the base category split, they are shown to induce weak bimonads (in four symmetric ways). As a consequence, they have four separable Frobenius base (co)monoids, two in each of the underlying monoidal categories. Hopf modules over weak bimonoids are defined by weakly lifting the induced comonad to the EilenbergMoore category of the induced monad. Making appropriate assumptions on the duoidal category in question, the fundamental theorem of Hopf modules is proven which says that the category of modules over one of the base monoids is equivalent to the category of Hopf modules if and only if a Galois-type comonad morphism is an isomorphism.
\end{abstract}

\section{INTRODUCTION}

Weak bialgebras were introduced first in the symmetric monoidal category of (finite dimensional) vector spaces [11, 20, 10]. They were generalized to braided monoidal categories (with split idempotents) in [22, 2]. The characteristic feature of weak bialgebras is the behavior of the category of their (co)modules. Similarly to usual, non-weak bialgebras, these categories are monoidal. However, as a 'weak' feature, the monoidal structure is different from that in the base category. That is to say, the forgetful functor is no longer strict monoidal as in the case of non-weak bialgebras, but it possesses a so-called 'separable Frobenius' monoidal structure 28. This means that it is both monoidal and opmonoidal but the morphisms which are responsible for these structures, are not mutually inverses of each other. The binary part of the monoidal structure only provides a left inverse of the binary part of the opmonoidal structure and some compatibility conditions reminiscent to those between the multiplication and the comultiplication of a Frobenius algebra hold. This property of the forgetful functor provides the basis of a generalization of weak bialgebras beyond braided monoidal base categories; to so-called 'weak bimonads' in [8].

Weak bimonads are monads on a monoidal category whose idempotent morphisms split, equipped with the additional structures that are equivalent to their Eilenberg-Moore category being monoidal with a forgetful functor possessing a separable Frobenius monoidal structure. The separable Frobenius monoidal forgetful functor takes the monoidal unit of the Eilenberg-Moore category to a separable Frobenius monoid in the base category [16] which is regarded as the 'base monoid' of the

Date: 2013 June.

2010 Mathematics Subject Classification. 16T05, 18D10, 18C15, 18C20.

Key words and phrases. duoidal category, weak bimonad, weak lifting, weak bimonoid, Hopf module.

Y.Y. Chen participated in this research as a fellow of the Hungarian Scholarship Board. Her work was supported also by the Innovative Project of Jiangsu province for Graduate Cultivation. 
weak bimonad in question. The monoidal structure of the Eilenberg-Moore category is given in fact by the module tensor product over the base monoid.

Braided monoidal categories were generalized to so-called 'duoidal categories' in [1] (where they were termed '2-monoidal categories'). These are categories carrying two, possibly different monoidal structures. The monoidal structures are required to be compatible in the sense that the functors and natural transformations defining the first monoidal structure, are opmonoidal with respect to the second monoidal structure. Equivalently, the functors and natural transformations defining the second monoidal structure, are monoidal with respect to the first monoidal structure (for more details see Section 1.1). Also bimonoids in duoidal categories were defined in [1]. These are objects which are monoids with respect to the first monoidal product $\circ$ and comonoids with respect to the second monoidal product $\bullet$. The compatibility axioms are formulated in terms of the coherence morphisms between the monoidal structures. These bimonoids generalize bialgebras in braided monoidal categories in such a way that their categories of modules (respectively, comodules) are still monoidal via the monoidal product $\bullet$ (respectively, o) lifted from the base category. In other words, they induce 'bimonads' (termed 'Hopf monads' in [18]) with respect to • (respectively, 'bicomonads' with respect to o), see [5].

The first aim of this paper is to find a common generalization of weak bimonoids in braided monoidal categories and of bimonoids in duoidal categories. So we consider an object in a duoidal category, which is a monoid with respect to the first monoidal structure and a comonoid with respect to the second monoidal structure. We look for compatibility conditions between them which imply the expected behavior of the (co)module categories: their monoidality via the (co)module tensor product over some separable Frobenius base monoid. The proposed axioms of what we call a weak bimonoid are presented in Section 2 where we also study their behavior under the various duality transformations in a duoidal category. As a main result, a weak bimonoid in a duoidal category in which idempotent morphisms split, is shown to induce four weak bi(co)monads (two on each of the underlying monoidal categories). Corresponding to the four induced weak bi(co)monads, there are four associated 'base objects' (two in each of the underlying monoidal categories), all of them carrying the structure of separable Frobenius monoid. Their properties are investigated in Section 3 and their relations to each other are studied in Section 4

Associated to any bimonoid in a duoidal category, there is a mixed distributive law (in the sense of [4, also known as an 'entwining structure', see [12]) between the induced monad and comonad. Its mixed modules are known as 'Hopf modules' 7. In order to describe Hopf modules over weak bialgebras (even over a field), however, mixed distributive laws had to be generalized to 'weak mixed distributive laws' in [14 (where they were termed 'weak entwining structures'). In Section 5 we construct a weak mixed distributive law between the monad and the comonad induced by a weak bimonoid in a duoidal category. Hopf modules are defined as its mixed modules. Applying the theory of weak liftings in [6], we also construct a (comparison) functor from the category of modules over one of the base monoids to the category of Hopf modules. In Section 6 we prove the fundamental theorem of Hopf modules: Under appropriate assumptions on the duoidal category in question, we show that this comparison functor is an equivalence if and only if a canonical Galoistype comonad morphism is an isomorphism. Recall that these equivalent properties distinguish 
weak Hopf algebras between weak bialgebras [13, Section 36.16]. In contrast to [7 - where in our study of non-weak bimonoids we used directly Beck's theory [4 to construct the inverse of the comparison functor - here we take a shorter route. The proof in Section 6 is based on a recent result due to Mesablishvili and Wisbauer in [17] about the properties of a functor occurring in the factorization of some separable left adjoint functor on a category whose idempotent morphisms split.

\section{Preliminaries}

1.1. Duoidal categories. In this section we recall from [1] some information about so-called duoidal (also known as 2-monoidal) categories. The occurring monoidal structures are not assumed to be strict. However, for brevity, we omit explicitly denoting the associator and the unitors. Throughout, the composition of any morphisms $\varphi$ and $\psi$ is denoted by $\varphi . \psi$.

Definition 1.1. [1, Definition 6.1] A duoidal category is a category $\mathcal{M}$ equipped with two monoidal products $\circ$ and $\bullet$ with respective units $I$ and $J$, along with morphisms

$$
I \stackrel{\delta}{\longrightarrow} I \bullet I, \quad J \circ J \stackrel{\varpi}{\longrightarrow} J, \quad I \stackrel{\tau}{\longrightarrow} J,
$$

and, for all objects $A, B, C, D$ in $\mathcal{M}$, a morphism

$$
\zeta_{A, B, C, D}:(A \bullet B) \circ(C \bullet D) \rightarrow(A \circ C) \bullet(B \circ D),
$$

called the interchange law, which is natural in all of the four occurring objects. These morphisms are required to obey the axioms below.

Compatibility of the units. The monoidal units $I$ and $J$ are compatible in the sense that

$$
(J, \varpi, \tau) \text { is a monoid in }(\mathcal{M}, \circ, I) \text { and }(I, \delta, \tau) \text { is a comonoid in }(\mathcal{M}, \bullet, J) \text {. }
$$

Associativity. For all objects $A, B, C, D, E, F$ in $\mathcal{M}$, the following diagrams commute.

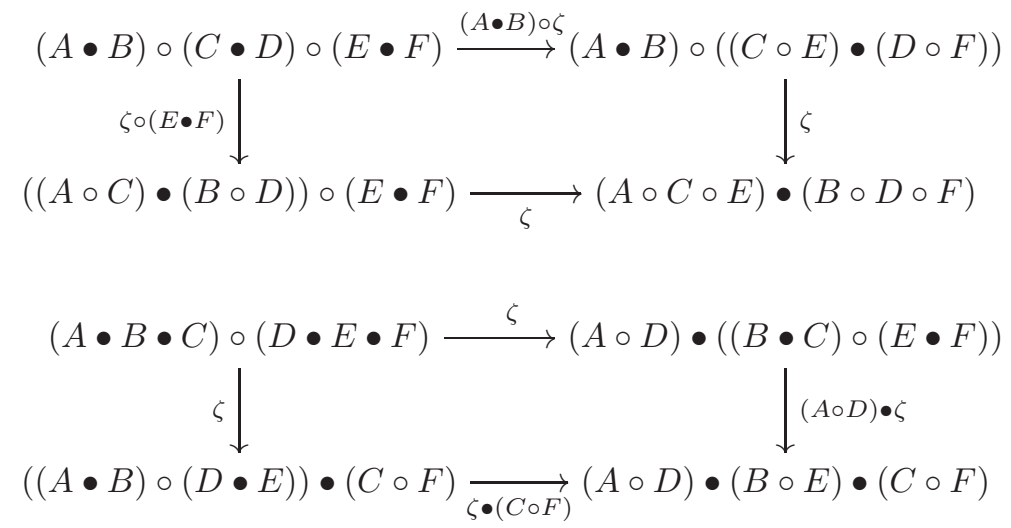

Unitality. For all objects $A, B$ in $\mathcal{M}$, the following diagrams commute.
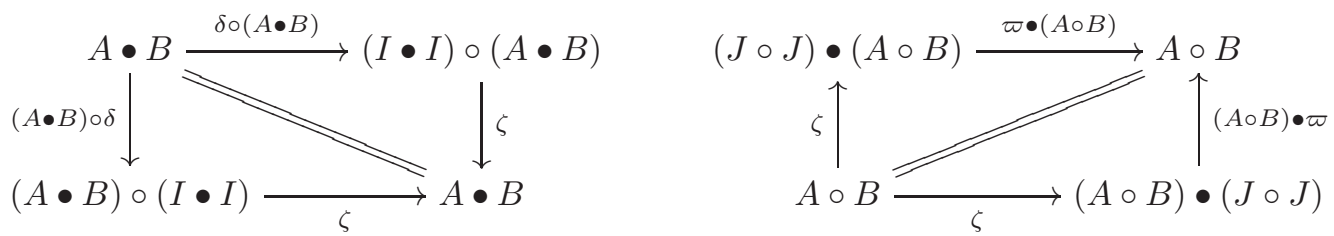
The simplest examples of duoidal categories are braided monoidal categories. In this case, both monoidal products coincide and the interchange law is induced by the braiding, see [1, Section 6.3].

In any duoidal category, for any objects $A$ and $B$, diagrams of the type

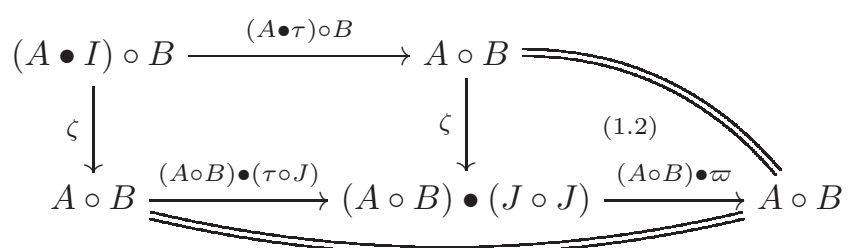

commute, see [1, Proposition 6.8].

1.2. Weak bimonads. The modules over a weak bialgebra (say, over a field) constitute a monoidal category. However, the monoidal product is different from the tensor product of vector spaces. This behavior of the category of modules was the basis of the generalization of weak bialgebras to weak bimonads in 8 .

Weak bimonads on a monoidal category (with monoidal product $\otimes$ and monoidal unit $K$ ) were defined in [8, Definition 1.3] as monads equipped with a monoidal structure of their EilenbergMoore category of modules (or algebras) and a separable Frobenius monoidal structure (in the sense of [28]) of the forgetful functor to the base category. Whenever idempotent morphisms in the base category split, this definition turns out to be equivalent to an opmonoidal structure $\left(T_{2}: T(-\otimes-) \rightarrow T(-) \otimes T(-), T_{0}: T K \rightarrow K\right)$ subject to five compatibility axioms in [8, Theorem 1.5] with the monad structure $\left(\mu: T^{2} \rightarrow T, \eta:\right.$ id $\left.\rightarrow T\right)$.

Recall from [8, proof of Proposition 1.11] that if $T$ is a weak bimonad on a monoidal category in which idempotent morphisms split, then for any $T$-modules $(F, \varphi)$ and $(G, \gamma)$ there is an idempotent morphism

$$
\chi_{F, G}: F \otimes G \stackrel{\eta_{F \otimes G}}{\longrightarrow} T(F \otimes G) \stackrel{T_{2}}{\longrightarrow} T F \otimes T G \stackrel{\varphi \otimes \gamma}{\longrightarrow} F \otimes G
$$

which is natural in $(F, \varphi)$ and $(G, \gamma)$. The monoidal product of the $T$-modules $(F, \varphi)$ and $(G, \gamma)$ is the object through which this idempotent morphism splits. Also the monoidal unit $R$ in the category of $T$-modules is obtained by splitting an idempotent morphism

$$
\sqcap: T K \stackrel{\eta T K}{\longrightarrow} T^{2} K \stackrel{T_{2}}{\longrightarrow} T K \otimes T^{2} K \stackrel{T K \otimes \mu_{K}}{\longrightarrow} T K \otimes T K \stackrel{T K \otimes T_{0}}{\longrightarrow} T K
$$

as $\sqcap=T K-\pi \rightarrow R \succ \iota \rightarrow T K$. By unitality of the monad $T$ and counitality of its opmonoidal structure,

$$
\sqcap . \eta K=\eta K \quad \text { and } \quad T_{0} \cdot \sqcap=T_{0} .
$$

By [8, eq. (1.10)], the morphism $\sqcap$ in (1.5) renders commutative

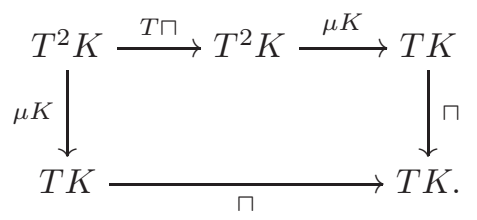

The $T$-action on $R$ is $\pi \cdot \mu_{K} \cdot T \iota$ (so that $\pi$ is a morphism of $T$-modules by (1.7)). Moreover, $R$ carries the structure of a separable Frobenius monoid in the base category (with multiplication 
and comultiplication obtained by the splitting $\chi_{R, R}=R \otimes R-\mu^{R} \rightarrow R \succ \Delta^{R} \rightarrow R \otimes R$ of (1.4), see [8, eqs. (2.1)-(2.2)]); and the monoidal product of $T$-modules turns out to be a module tensor product over $R$. By the second equality in (1.6) and by commutativity of

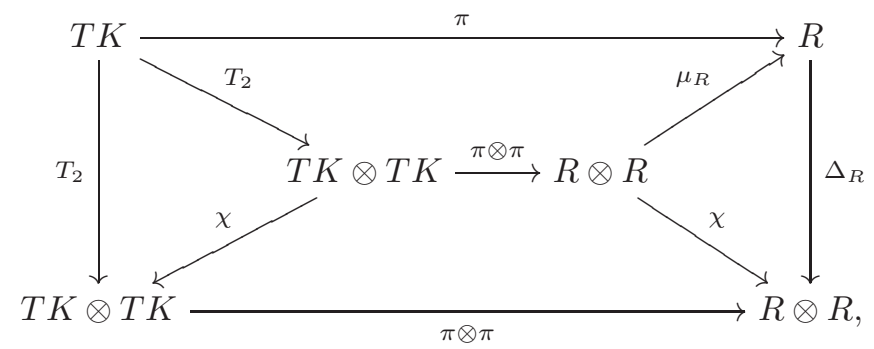

$\pi: T K \rightarrow R$ is a morphism of comonoids. (The region on the left commutes by the weak bimonad axiom [8, eq. (1.7)] and unitality of the monad $T$; and the region at the top commutes by the naturality of $\chi$, counitality of the opmonoidal functor $T$ and the second equality in (1.6) and $T_{2}=\chi \cdot T_{2}$ again, taking into account the explicit expression of $\mu_{R}$ in $[8$, eq. (2.1)].)

Whenever $T$ is a weak bimonad on a monoidal category (whose idempotent morphisms split), it is a weak bimonad (with the same monad, and opmonoidal structures) on the opposite monoidal category as well. Corresponding to this latter weak bimonad on the opposite monoidal category, there is a symmetric counterpart

$$
\bar{\Pi}: T K \stackrel{\eta T K}{\longrightarrow} T^{2} K \stackrel{T_{2}}{\longrightarrow} T^{2} K \otimes T K \stackrel{\mu K \otimes T K}{\longrightarrow} T K \otimes T K \stackrel{T_{0} \otimes T K}{\longrightarrow} T K
$$

of $\sqcap$ in (1.5). It is shown in [8, page 12] that $\sqcap \cdot \bar{\Pi}=\sqcap$. Symmetrically, $\bar{\Pi} . \sqcap=\bar{\Pi}$. Hence taking the splittings

$$
\Pi=T K \stackrel{\pi}{\longrightarrow} R \stackrel{\iota}{\longleftrightarrow} T K \quad \text { and } \bar{\Pi}=T K \stackrel{\bar{\pi}}{\longrightarrow} \bar{R} \stackrel{\bar{\imath}}{\longrightarrow} T K
$$

of these idempotent morphisms, we obtain mutually inverse isomorphisms

$$
R \stackrel{\iota}{\longrightarrow} T K \stackrel{\bar{\pi}}{\longrightarrow} \bar{R} \quad \text { and } \quad \bar{R} \stackrel{\bar{\imath}}{\longrightarrow} T K \stackrel{\pi}{\longrightarrow} R .
$$

Together with the natural transformation (1.4), they render commutative

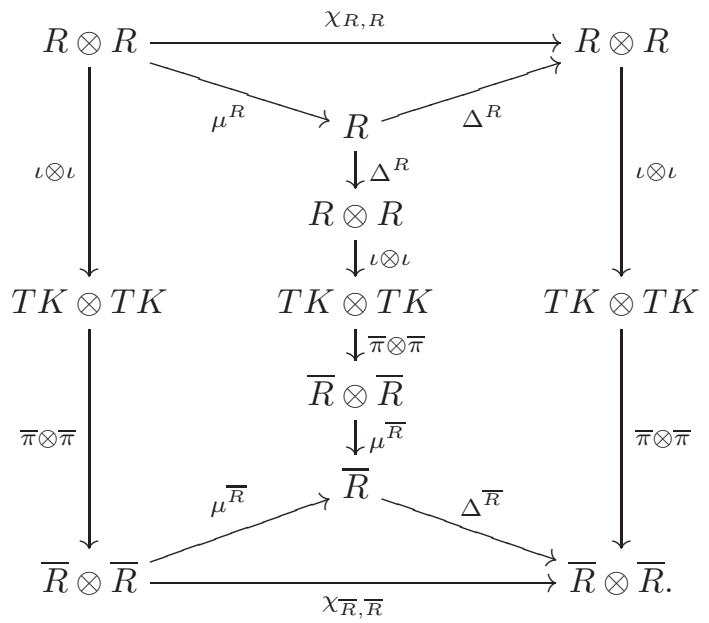


The vertical arrow $R \rightarrow \bar{R}$ at the middle obeys

$$
\begin{aligned}
\mu^{\bar{R}} \cdot(\bar{\pi} \otimes \bar{\pi}) \cdot(\iota \otimes \iota) \cdot \Delta^{R} \cdot \pi & =\mu^{\bar{R}} \cdot(\bar{\pi} \otimes \bar{\pi}) \cdot(\iota \otimes \iota) \cdot(\pi \otimes \pi) \cdot \Delta^{T K} \\
& =\mu^{\bar{R}} \cdot(\bar{\pi} \otimes \bar{\pi}) \cdot \Delta^{T K}=\mu^{\bar{R}} \cdot \Delta^{\bar{R}} \cdot \bar{\pi}=\bar{\pi}=\bar{\pi} \cdot \iota \cdot \pi .
\end{aligned}
$$

In the first and the third equalities we used that $\pi$ and $\bar{\pi}$ are comultiplicative. In the second and the last equalities we used that $\bar{\Pi} . \sqcap=\bar{\Pi}$ hence $\bar{\pi} \cdot \iota \cdot \pi=\bar{\pi}$. In the fourth equality we used that $\mu^{\bar{R}} \cdot \Delta^{\bar{R}}$ is the identity (that is, the separability of $\bar{R}$ ). Since $\pi$ is an epimorphism, this proves that the vertical arrow $R \rightarrow \bar{R}$ at the middle of the diagram is equal to the isomorphism in (1.8), hence it is both multiplicative and comultiplicative. It is also unital and counital by (1.6) and the explicit expressions of the unit and the counit in [8, (2.1) and (2.2)]. This proves that (1.8) is an isomorphism of monoids and comonoids.

The forgetful functor, from the Eilenberg-Moore category of $T$-modules to the base category, factorizes through the category of $R$-bimodules [8, page 14]. That is, any $T$-module carries canonical (commuting) left and right $R$-actions and any morphism of $T$-modules is compatible with them. For example, (1.4) is a morphism of $T$-modules (hence so are its splitting mono- and epimorphisms), thus it is a morphism of $R$-bimodules.

In the diagram

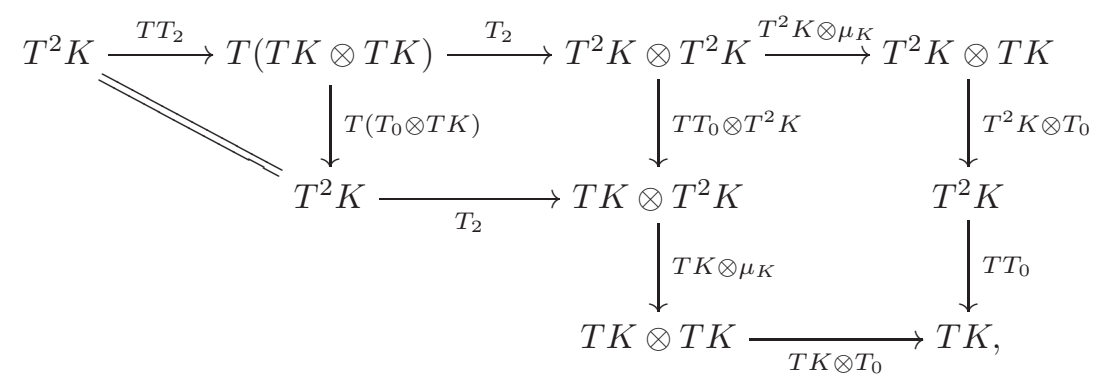

the triangle on the left commutes by counitality of the opmonoidal structure, and both regions on the right commute by naturality of $T_{2}$ and functoriality of the monoidal product. This allows to write $\sqcap$ in the alternative form

$$
T K \stackrel{\eta T K}{\longrightarrow} T^{2} K \stackrel{T T_{2}}{\longrightarrow} T(T K \otimes T K) \stackrel{T_{2}}{\longrightarrow} T^{2} K \otimes T^{2} K \stackrel{T^{2} K \otimes \mu_{K}}{\longrightarrow} T^{2} K \otimes T K \stackrel{T T_{0} \otimes T_{0}}{\longrightarrow} T K .
$$

\section{THE WEAK BIMONOID AXIOMS}

In this section we introduce the central notion of the paper: weak bimonoid in a duoidal category. It is shown to provide a common generalization of bimonoids in duoidal categories [1, Definition 6.25], and of weak bimonoids in braided monoidal categories [22. If idempotent morphisms in the base category split, any weak bimonoid is proven to induce four bi(co)monads.

Definition 2.1. A right weak bimonoid in a duoidal category $\mathcal{M}$ is an object $A$ equipped with a monoid structure $(A \circ A \stackrel{\mu}{\rightarrow} A, I \stackrel{\eta}{\rightarrow} A)$ in $(\mathcal{M}, \circ)$ and a comonoid structure $(A \stackrel{\Delta}{\rightarrow} A \bullet A, A \stackrel{\varepsilon}{\rightarrow} J)$ in $(\mathcal{M}, \bullet)$, subject to the five compatibility axioms listed below. 
In the same way as for a bimonoid, the multiplication is required to be comultiplicative, equivalently, the comultiplication is required to be multiplicative:

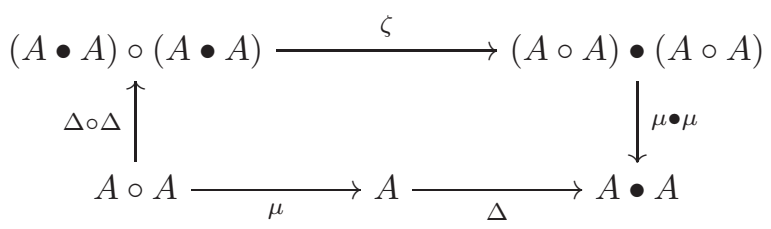

Comultiplicativity of the unit is replaced by two weaker conditions

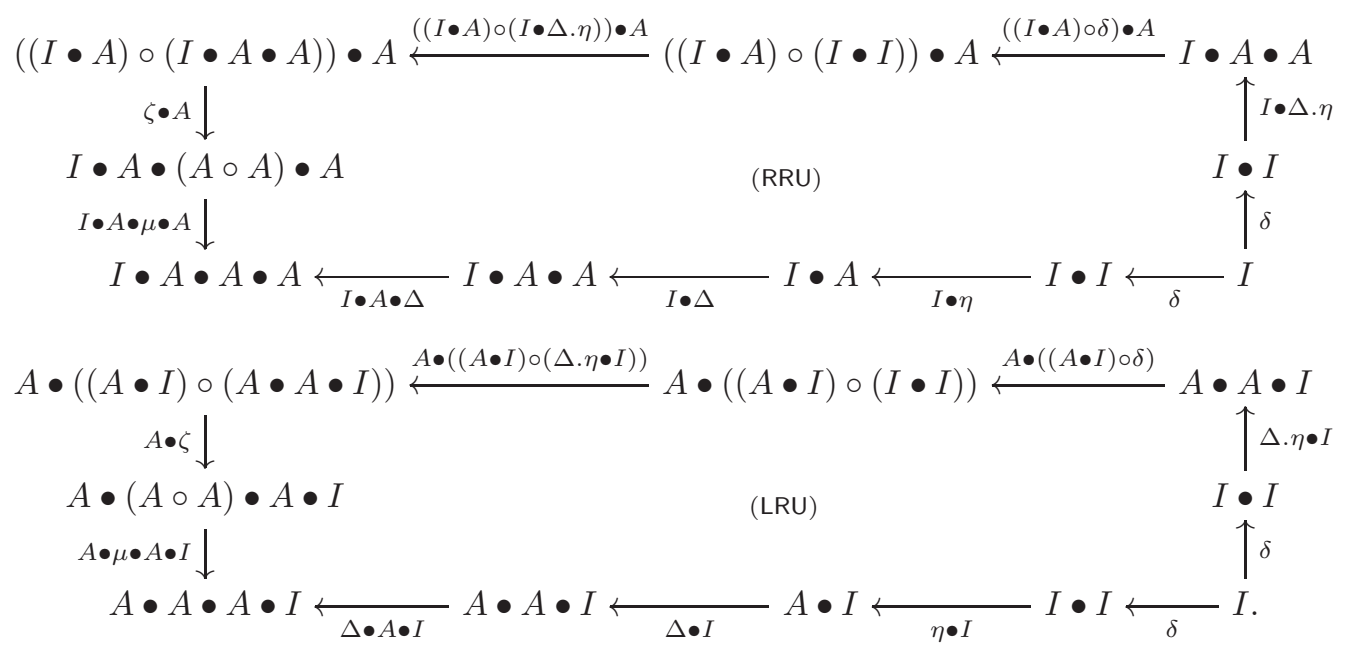

Multiplicativity of the counit is replaced by two weaker conditions

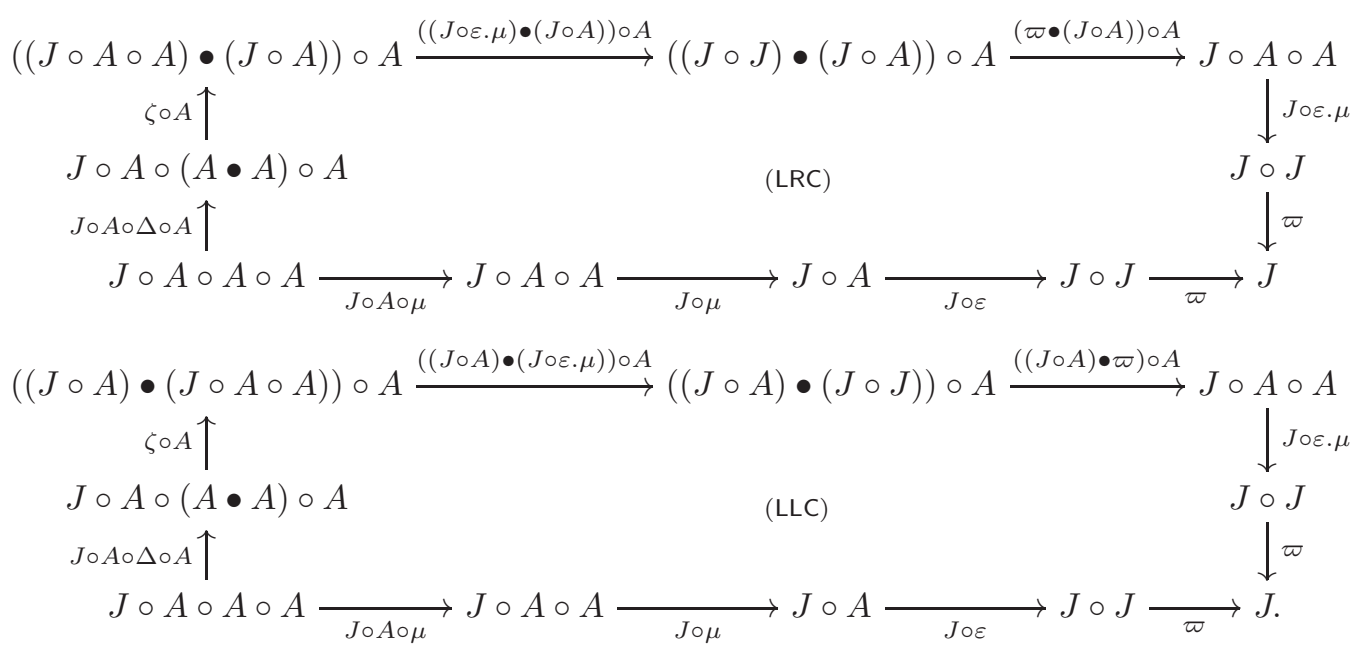

A duoidal structure can be twisted in three different ways, see [1, Section 6.1.2]. One can replace any of the monoidal products by the opposite one; and as a third possibility, one can interchange the roles of both monoidal products and change simultaneously the composition of morphisms to the opposite one. That is, associated to any duoidal category $(\mathcal{M}, \circ, \bullet)$, there are seven more duoidal categories $\left(\mathcal{M}, \circ^{\text {op }}, \bullet\right),\left(\mathcal{M}, \circ, \bullet^{\text {op }}\right),\left(\mathcal{M}, \circ^{\text {op }}, \bullet^{\text {op }}\right),\left(\mathcal{M}^{\text {op }}, \bullet, \circ\right),\left(\mathcal{M}^{\text {op }}, \bullet, \circ^{\text {op }}\right),\left(\mathcal{M}^{\text {op }}, \bullet^{\text {op }}, \circ\right)$, $\left(\mathcal{M}^{\mathrm{op}}, \bullet^{\mathrm{op}}, \mathrm{o}^{\mathrm{op}}\right)$. 
Alternatively, changing the passive and active points of view, to any diagram in $\mathcal{M}$ one can apply the transformations $\circ \leftrightarrow \circ^{\text {op }}, \bullet \leftrightarrow \bullet$ op or $\left(\circ \leftrightarrow \bullet, \mathcal{M} \leftrightarrow \mathcal{M}^{\text {op }}\right)$ — which will be denoted by $\circ$, • and $*$, respectively - and any of their composites. Clearly, the diagrams defining a monoid in $(\mathcal{M}, \circ)$ are invariant under the transformation $\circ$ or $\bullet$; and they are taken by $*$ to the diagrams defining a comonoid in $(\mathcal{M}, \bullet)$. Symmetrically, the diagrams defining a comonoid in $(\mathcal{M}, \bullet)$ are invariant under the transformation $\circ$ or $\bullet$; and they are taken by $*$ to the diagrams defining a monoid in $(\mathcal{M}, \circ)$. What is more, any of the transformations $\circ$, $\bullet$ and $*$ leaves invariant the set of diagrams defining a bimonoid in $(\mathcal{M}, \circ, \bullet)$.

Our next task is to study the behavior of the weak bimonoid axioms (RRU), (LRU), (LLC), (LRC) in Definition 2.1 under these transformations. The set of these diagrams is closed under the action of $\bullet$ (which connects (RRU) with (LRU), and (LLC) with (LRC)). It is not closed, however, under the action of the other two transformations. We obtain a set of diagrams which are closed under all transformations $\circ$, $\bullet$ and $*$, if we add the images under $\circ$. We denote the images of $(\mathrm{RRU}),(\mathrm{LRU}),(\mathrm{LLC}),(\mathrm{LRC})$ under $\circ$ by (RLU), (LLU), (RLC), (RRC), respectively. Explicitly, they read as

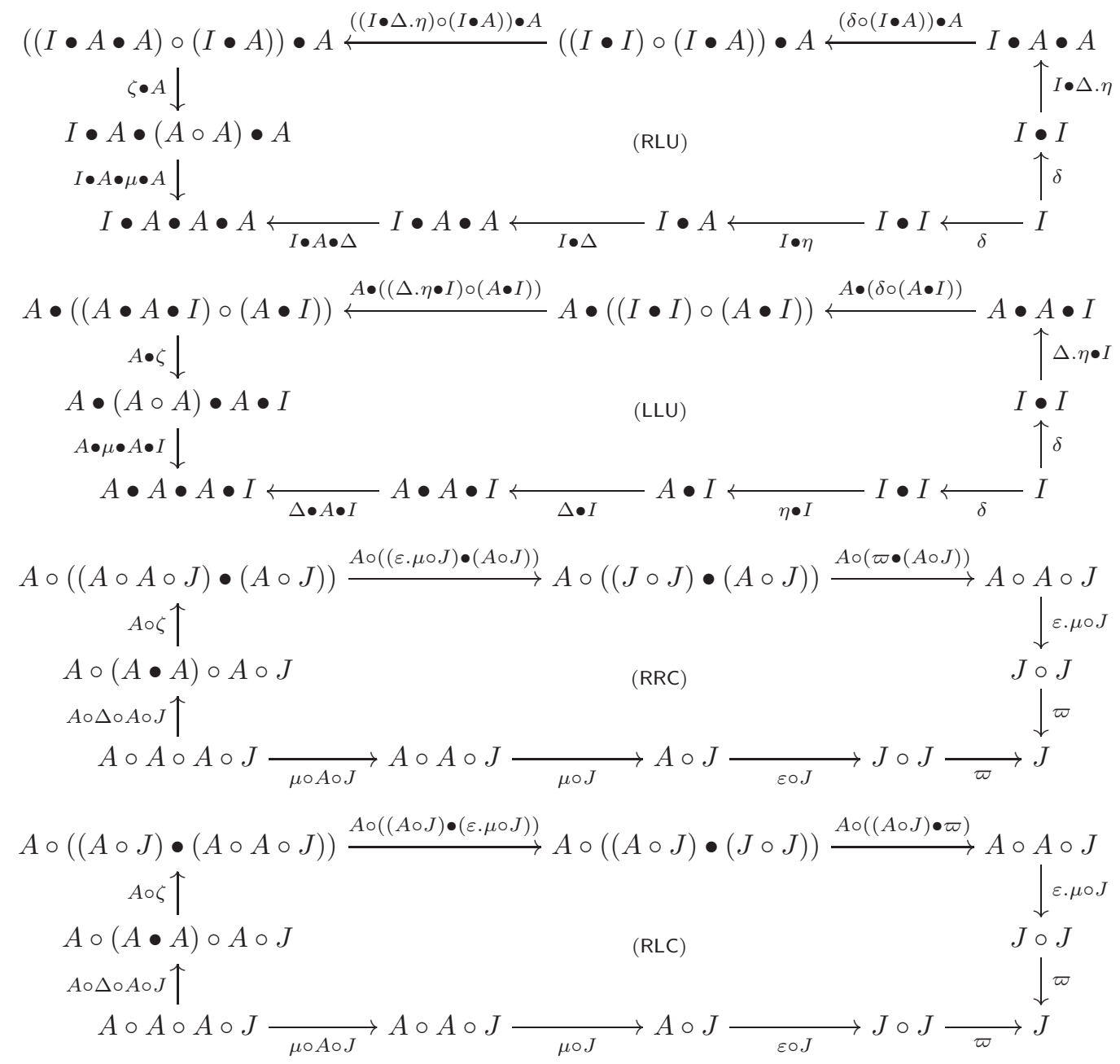


We obtain the following picture of the actions on these diagrams.

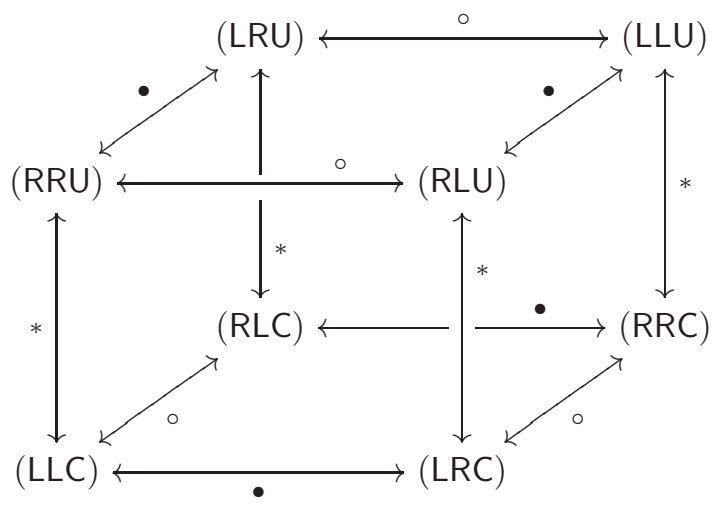

In the next two propositions we present two large classes of examples of right weak bimonoids in duoidal categories.

Proposition 2.2. Any bimonoid in a duoidal category satisfies the right weak bimonoid axioms in Definition 2.1. That is, right weak bimonoid provides a generalization of bimonoid in a duoidal category.

Proof. If $A$ is a bimonoid, then the multiplication is comultiplicative by assumption. Since $\Delta . \eta=$ $(\eta \bullet \eta) . \delta$, (RRU) holds by commutativity of

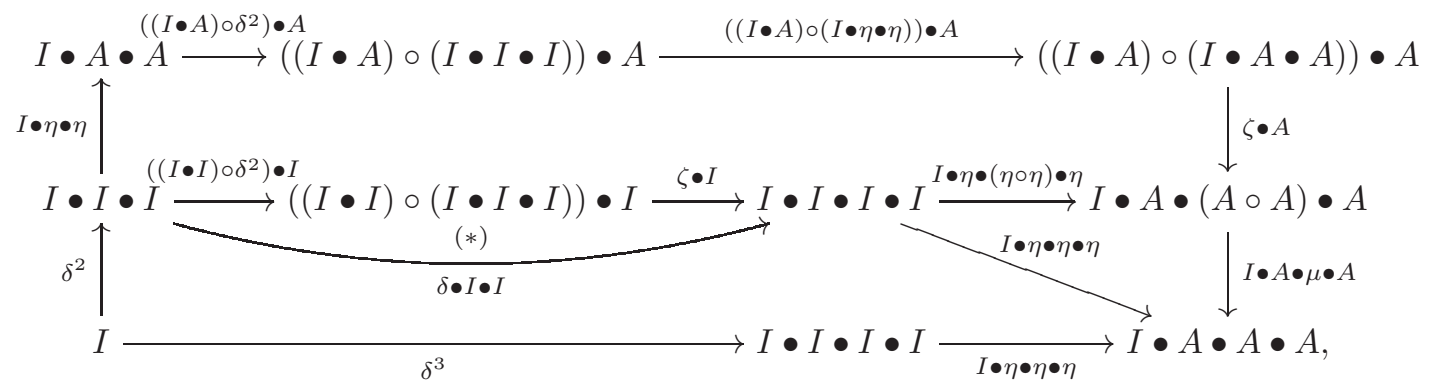

where, as usual, we denoted $\delta^{2}=(I \bullet \delta) . \delta=(\delta \bullet I) . \delta, \delta^{3}=\left(I \bullet \delta^{2}\right) . \delta=\left(\delta^{2} \bullet I\right) . \delta$, and so on. The top region commutes by functoriality of both monoidal products and naturality of $\zeta$. The triangle at the bottom right commutes by the unitality of $\mu$. The region marked by $(*)$ commutes by naturality of $\zeta$ and a unitality axiom in (1.2). The bottom region commutes by the coassociativity of $\delta$.

Since the bimonoid axioms are invariant under any of the transformations $\circ$, $\bullet$ and $*$, all other axioms of right weak bimonoid in Definition 2.1 hold by symmetry.

Proposition 2.3. Regard any braided monoidal category $(\mathcal{M}, \otimes, I, \sigma)$ as a duoidal category with coinciding monoidal structures $(\otimes, I)$ and the interchange law provided by the braiding $\sigma$. Then a weak bimonoid in the sense of 22 is the same as a right weak bimonoid in Definition 2.1. In this way, right weak bimonoids in duoidal categories provide a generalization also of weak bimonoids in braided monoidal categories.

Proof. The multiplication is comultiplicative by definition, for any weak bimonoid in the sense of 22 and also in the sense of Definition 2.1. Axiom (RRU) is equivalent to commutativity of the 
exterior of the following diagram; and axiom (w.2) in [22] - expressing weak comultiplicativity of the unit — is equivalent to commutativity of the bottom region in

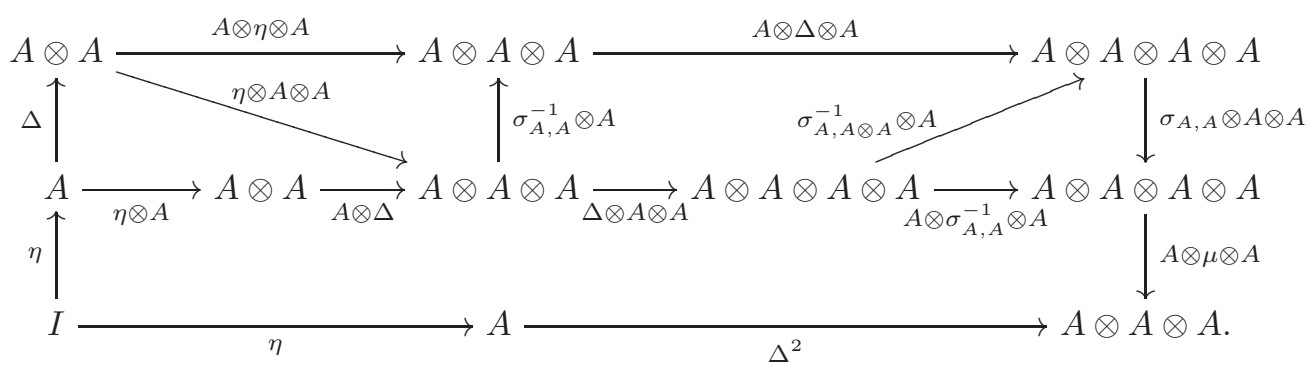

So they are equivalent by commutativity of all regions at the top: The leftmost region commutes by functoriality of the monoidal product and the remaining regions commute by coherence and naturality of the braiding.

The remaining three axioms are shown to be pairwise equivalent symmetrically.

As a most important justification of Definition 2.1] right weak bimonoids induce weak bimonads (in the sense of 8 ). The proof of this fact starts with the following.

Lemma 2.4. For a right weak bimonoid $A$, and any objects $X$ and $Y$ in a duoidal category $(\mathcal{M}, \circ, \bullet)$, consider the morphism

$$
\kappa_{X, Y}:((J \circ A) \bullet X) \circ(A \bullet Y) \stackrel{\zeta}{\longrightarrow}(J \circ A \circ A) \bullet(X \circ Y) \stackrel{(J \circ \varepsilon \cdot \mu) \bullet(X \circ Y)}{\longrightarrow}(J \circ J) \bullet(X \circ Y) \stackrel{\varpi \bullet(X \circ Y)}{\longrightarrow} X \circ Y .
$$

The following assertions hold.

(1) $\kappa$ is natural both in $X$ and $Y$.

(2) The following diagram commutes, for any objects $X, Y, Z$ in $\mathcal{M}$.

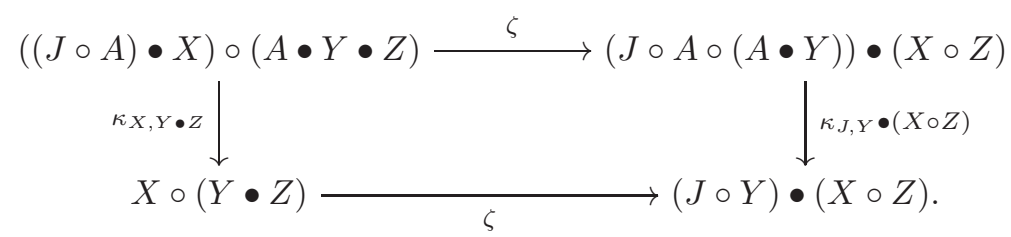

(3) The following diagram commutes, for any objects $X, Y$ in $\mathcal{M}$.

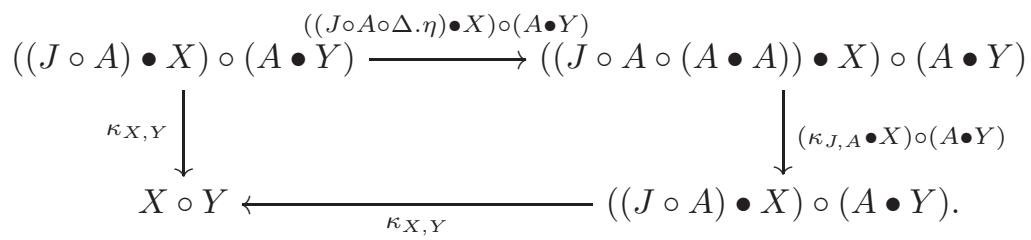

Proof. (1) is evident by naturality of $\zeta$ and functoriality of both monoidal products.

(2) follows easily by one of the associativity conditions in (1.1) and functoriality of $\bullet$.

(3) is proven in the Appendix, on page 27. Commutativity of the region labelled by (LRC) in the diagram on page 27 follows by precomposing both equal paths in (LRC) with $\tau \circ A \circ \eta \circ A$. The undecorated regions commute by naturality and the associativity of $\varpi$. 
Theorem 2.5. Any right weak bimonoid $A$ in a duoidal category $(\mathcal{M}, \circ, \bullet)$ in which idempotent morphisms split, induces a weak bimonad $(-) \circ A$ on $(\mathcal{M}, \bullet)$.

Proof. The multiplication and the unit of the monad are induced by the multiplication and the unit of the monoid $A$, respectively. The binary part of the opmonoidal structure is given by

$$
\left(M \bullet M^{\prime}\right) \circ A \stackrel{\left(M \bullet M^{\prime}\right) \circ \Delta}{\longrightarrow}\left(M \bullet M^{\prime}\right) \circ(A \bullet A) \stackrel{\zeta}{\longrightarrow}(M \circ A) \bullet\left(M^{\prime} \circ A\right),
$$

for all objects $M, M^{\prime}$ in $\mathcal{M}$. The nullary part is provided by $J \circ A \stackrel{J \circ \varepsilon}{\longrightarrow} J \circ J \stackrel{\varpi}{\longrightarrow} J$. This equips $(-) \circ A$ with the structure of an opmonoidal functor indeed: coassociativity follows from the coassociativity of $\Delta$, the associativity axioms (1.1) in a duoidal category and naturality of $\zeta$; and counitality follows by the counitality of $\Delta$, the unitality axioms (1.2) in a duoidal category and naturality of $\zeta$ again. (The proof of this is identical to the non-weak case.)

We use the equivalent conditions in [8, Theorem 1.5] to show that $(-) \circ A$ is a weak bimonad. First, [8, eq. (1.7)] holds true by one of the associativity axioms of a duoidal category in (1.1), the compatibility condition (2.1), naturality of $\zeta$ and functoriality of $\circ$ (this proof is identical to the non-weak case). Verification of [8, eq. (1.5)], for any objects $X, Y$ and $Z$ of $\mathcal{M}$, can be found in the Appendix, on page 28. Commutativity of the region labelled by (RRU) in the diagram on page 28 follows by postcomposing both sides of axiom (RRU) with $\tau \bullet A \bullet A \bullet A$. The undecorated regions commute by naturality. Finally, [8, eq. (1.4)] is proven to hold, for any object $X$ in $\mathcal{M}$, in the Appendix, on page 29. The proof makes use of Lemma 2.4. Commutativity of the region labelled by (LRU) in the diagram on page 29, follows by postcomposing both sides of (LRU) with $A \bullet A \bullet A \bullet \tau$. The vertical arrow on the right-hand-side of the diagram on page 29 is equal to the identity morphism by one of the unitality axioms in (1.2). The remaining diagrams in [8, Theorem 1.5] can be obtained from [8, eqs. (1.4) and (1.5)] replacing • by its opposite. Hence they hold true by symmetry.

The transformations $\circ, \bullet$ and $*$ relate also the functors induced by any object $A$, as depicted in the diagram

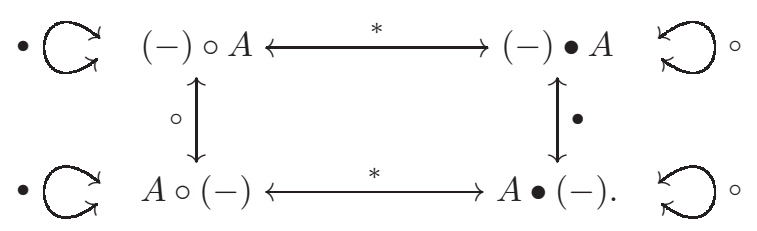

Hence by symmetry considerations, from Theorem 2.5 we obtain the following. (By a weak bicomonad below, we mean a weak bimonad on the opposite category.)

Corollary 2.6. Let $\mathcal{M}$ be a duoidal category with monoidal products $\circ$ and $\bullet$, such that idempotent morphisms in $\mathcal{M}$ split. Let $A$ be an object of $\mathcal{M}$ which carries the structures of a monoid wrt $\circ$ and a comonoid wrt $\bullet$. Assume that (2.1) holds true.

(1) If (RLU), (LLU), (RLC), and (RRC) hold, then $A \circ(-)$ is a weak bimonad on $(\mathcal{M}, \bullet)$.

(2) If (LLC), (RLC), (RRU) and (RLU) hold, then $(-) \bullet A$ is a weak bicomonad on $(\mathcal{M}, \circ)$.

(3) If (LRC), (RRC), (LRU) and (LLU) hold, then $A \bullet(-)$ is a weak bicomonad on $(\mathcal{M}, \circ)$. 
This motivates the following.

Definition 2.7. A left weak bimonoid in a duoidal category $(\mathcal{M}, \circ, \bullet)$ is an object $A$ which carries the structures of a monoid wrt $\circ$ and a comonoid wrt $\bullet$ such that (2.1) and the conditions (RLU), (LLU), (RLC), and (RRC) hold. We say that $A$ is a weak bimonoid if it is both a right weak bimonoid and a left weak bimonoid.

In a braided monoidal category — regarded as a duoidal category — all notions of right weak bimonoid, left weak bimonoid and weak bimonoid coincide. Bimonoids in duoidal categories also provide examples of both right weak bimonoids and left weak bimonoids (hence of weak bimonoids).

\section{The MONOIDAL CATEgORY OF MOdules}

In light of Theorem 2.5, we can apply all the information about weak bimonads in Section 1.2 to the weak bimonad $(-) \circ A$ on $(\mathcal{M}, \bullet)$, induced by a right weak bimonoid $A$ in a duoidal category $(\mathcal{M}, \circ, \bullet)$ in which idempotent morphisms split.

The monoidal product of any $(-) \circ A$-modules $(F, \varphi)$ and $(G, \gamma)$ is given by splitting the idempotent morphism (1.4), that is,

$$
\chi_{F, G}^{R}: F \bullet G \stackrel{(F \bullet G) \circ \Delta . \eta}{\longrightarrow}(F \bullet G) \circ(A \bullet A) \stackrel{\zeta}{\longrightarrow}(F \circ A) \bullet(G \circ A) \stackrel{\varphi \bullet \gamma}{\longrightarrow} F \bullet G .
$$

The monoidal unit of the category of $(-) \circ A$-modules — to be denoted by $R_{\circ}$ — is obtained by splitting the idempotent morphism (1.5), taking now the explicit form

$$
\Pi_{\circ}^{R}: J \circ A \stackrel{J \circ A \circ \Delta . \eta}{\longrightarrow} J \circ A \circ(A \bullet A) \stackrel{\zeta}{\rightarrow}(J \circ A) \bullet(J \circ A \circ A) \stackrel{(J \circ A) \bullet(J \circ \varepsilon . \mu)}{\longrightarrow}(J \circ A) \bullet(J \circ J) \stackrel{(J \circ A) \bullet \varpi}{\longrightarrow} J \circ A .
$$

By (1.10), the duoidal category axioms (1.1) and (1.2), by the counitality of $\Delta$, naturality of $\zeta$ and functoriality of $\circ, \Pi_{\circ}^{R}$ admits an equal form

$$
J \circ A \stackrel{J \circ A \circ \Delta . \eta}{\longrightarrow} J \circ A \circ(A \bullet A) \stackrel{J \circ \zeta}{\longrightarrow} J \circ((J \circ A) \bullet(A \circ A)) \stackrel{J \circ((J \circ A) \bullet \varepsilon . \mu)}{\longrightarrow} J \circ J \circ A \stackrel{\varpi \circ A}{\longrightarrow} J \circ A .
$$

For the splittings of $\sqcap_{\circ}^{R}$ and $\chi_{F, G}^{R}$, the notations
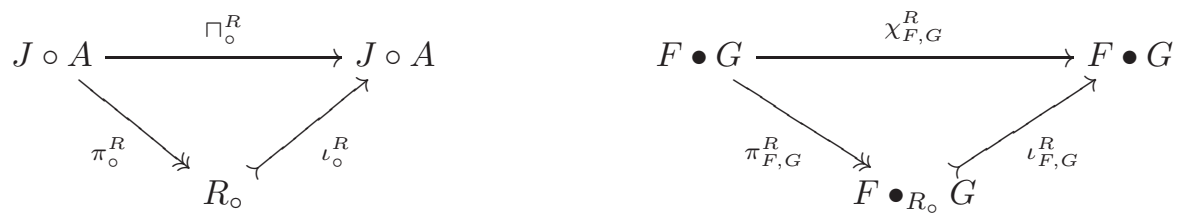
will be used. The retract $R_{\circ}$ is a separable Frobenius monoid in $(\mathcal{M}, \bullet)$, with multiplication, unit, comultiplication and counit as below, see [8, eqs. (2.1)-(2.2)].

$$
\begin{array}{ll}
\mu_{\circ}^{R}: & R_{\circ} \bullet R_{\circ} \stackrel{\chi^{R}}{\longrightarrow} R_{\circ} \bullet R_{\circ} \stackrel{R_{\circ} \bullet \iota_{\circ}^{R}}{\longrightarrow} R_{\circ} \bullet(J \circ A) \stackrel{R_{\circ} \bullet(J \circ \varepsilon)}{\longrightarrow} R_{\circ} \bullet(J \circ J) \stackrel{R_{\circ} \bullet \varpi}{\longrightarrow} R_{\circ}, \\
\eta_{\circ}^{R}: & J \circ A \stackrel{\pi_{\circ}^{R}}{\longrightarrow} R_{\circ}, \\
\Delta_{\circ}^{R}: & R_{\circ} \stackrel{R_{\circ} \bullet(J \circ \eta)}{\longrightarrow} R_{\circ} \bullet(J \circ A) \stackrel{R_{\circ} \bullet \pi_{\circ}^{R}}{\longrightarrow} R_{\circ} \bullet R_{\circ} \stackrel{\chi^{R}}{\longrightarrow} R_{\circ} \bullet R_{\circ}, \\
\varepsilon_{\circ}^{R}: \quad & R_{\circ} \stackrel{\iota_{\circ}^{R}}{\longrightarrow} J \circ A \stackrel{J \circ \varepsilon}{\longrightarrow} J \circ J \stackrel{\varpi}{\longrightarrow} J .
\end{array}
$$

By construction, $\pi_{\circ}^{R}: J \circ A \rightarrow R_{\circ}$ is a morphism of comonoids, hence it induces (left and right) $R_{\circ}$-coactions on $J \circ A$.

Lemma 3.1. For any right weak bimonoid $A$ in a duoidal category $(\mathcal{M}, \circ, \bullet)$ in which idempotent morphisms split, $\Pi_{\circ}^{R}: J \circ A \rightarrow J \circ A$ in (3.2) (and hence the epimorphism $\pi_{\circ}^{R}: J \circ A \rightarrow R_{\circ}$ and the monomorphism $\iota_{\circ}^{R}: R_{\circ} \rightarrow J \circ A$ in its splitting) are morphisms of right $R_{\circ}$-comodules.

Proof. The idempotent $\Pi_{\circ}^{R}: J \circ A \rightarrow J \circ A$ is a morphism of right $R_{\circ}$-comodules if and only if

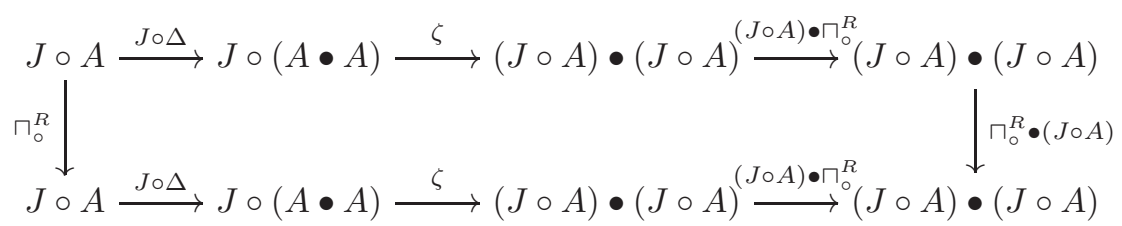

commutes. Using expression (3.2) of $\Pi_{0}^{R}$, the coassociativity of $\Delta$, naturality of $\zeta$ and functoriality of $\bullet$, the down-then-right path in (3.5) is checked to be equal to

$$
\begin{aligned}
& J \circ A \stackrel{J \circ A \circ \Delta^{2} . \eta}{\longrightarrow} J \circ A \circ(A \bullet A \bullet A) \stackrel{\zeta}{\longrightarrow}(J \circ(A \bullet A)) \bullet(J \circ A \circ A) \stackrel{\zeta \bullet(J \circ A \circ A)}{\longrightarrow} \\
& (J \circ A) \bullet(J \circ A) \bullet(J \circ A \circ A) \longrightarrow(J \circ A) \bullet(J \circ A) \bullet(J \circ J) \stackrel{(J \circ A) \bullet \sqcap_{\circ}^{R} \bullet \varpi}{\longrightarrow}(J \circ A) \bullet(J \circ A) .
\end{aligned}
$$

Hence by commutativity of the diagram on page 30 in the Appendix, it is equal to $(3.6)$

$$
\begin{aligned}
& J \circ A \circ \Delta . \eta \\
& J \circ A \rightarrow J \circ A \circ(A \bullet A) \stackrel{\zeta}{\rightarrow}(J \circ A) \bullet(J \circ A \circ A) \stackrel{(J \circ A) \bullet(J \circ \mu)}{\rightarrow}(J \circ A) \bullet(J \circ A) \stackrel{(J \circ A) \bullet \sqcap^{R}}{\rightarrow}(J \circ A) \bullet(J \circ A) .
\end{aligned}
$$

In the diagram on page 30, the region labelled by $(*)$ is identical to the commutative diagram on page 28 if we substitute in the latter one $X=Y=J$ and $Z=J \circ A$. On the other hand, also the right-then-down path in (3.5) is equal to (3.6) by commutativity of the diagram on page 31 in the Appendix. Commutativity of the region marked by $(*)$ in the diagram on page 31 follows by commutativity of the diagram on page 28 (substituting in it $X=J$ and $Y=Z=A$ ). The vertical path on the right hand side of the diagram on page 31 is equal to $\sqcap_{0}^{R} \bullet(J \circ A)$ by (3.3). This proves that $\Pi_{\circ}^{R}$ is a morphism of right $R_{\circ}$-comodules. Then so are its splitting mono- and epimorphisms with respect to the coaction

$$
R_{\circ} \stackrel{\iota_{\circ}^{R}}{\longrightarrow} J \circ A \stackrel{J \circ \Delta}{\longrightarrow} J \circ(A \bullet A) \stackrel{\zeta}{\longrightarrow}(J \circ A) \bullet(J \circ A) \stackrel{\pi_{\circ}^{R} \bullet \pi_{\circ}^{R}}{\longrightarrow} R_{\circ} \bullet R_{\circ}=\Delta_{\circ}^{R}
$$

on $R_{\circ}$, where the equality follows by using that $\pi_{\circ}^{R}$ is a morphism of comonoids. 
Lemma 3.2. For any right weak bimonoid $A$ in a duoidal category $(\mathcal{M}, \circ, \bullet)$ in which idempotent morphisms split, there is a morphism of comonoids $A \stackrel{\tau \circ A}{\longrightarrow} J \circ A \stackrel{\pi_{\circ}^{R}}{\longrightarrow} R_{\circ}$.

Proof. Since $\pi_{\circ}^{R}: J \circ A \rightarrow R_{\circ}$ is a morphism of comonoids, we only need to show that $\tau \circ A$ : $A \rightarrow J \circ A$ is so. Its counitality follows by the unitality of $\varpi$ and its comultiplicativity follows by commutativity of the following diagram (in which the middle region commutes by the counitality of $\delta)$.

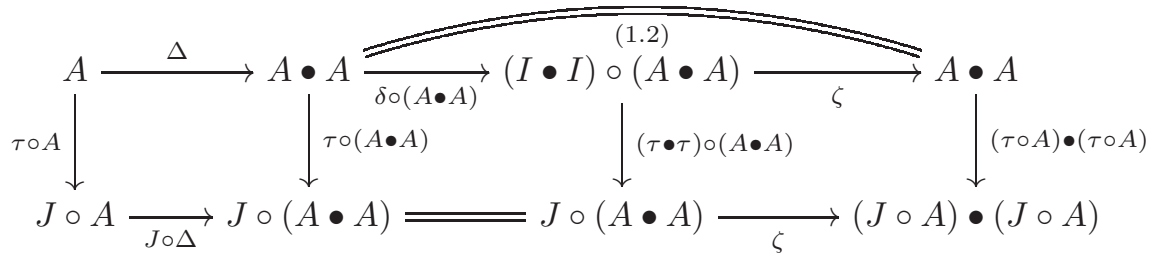

Lemma 3.3. For any right weak bimonoid $A$ in a duoidal category $(\mathcal{M}, \circ, \bullet)$ in which idempotent morphisms split, $R_{\circ}$ is a $J$-A bimodule.

Proof. Recall from [8, page 11] that $R_{\circ}$ is a right $A$-module via the action

$$
R_{\circ} \circ A \stackrel{\iota_{\circ}^{R} \circ A}{\longrightarrow} J \circ A \circ A \stackrel{J \circ \mu}{\longrightarrow} J \circ A \stackrel{\pi_{\circ}^{R}}{\longrightarrow} R_{\circ} .
$$

Symmetrically, it is a left $J$-module via

$$
J \circ R \circ \stackrel{J \circ \iota_{\circ}^{R}}{\longrightarrow} J \circ J \circ A \stackrel{\varpi \circ A}{\longrightarrow} J \circ A \stackrel{\pi_{\circ}^{R}}{\longrightarrow} R_{\circ} .
$$

Unitality of both actions is evident. They are also associative and commute with each other by the associativity of $\mu$ and of $\varpi$, together with (1.7) and the fact that $\Pi_{0}^{R}$ is a morphism of left $J$-modules (what follows from its form in (3.3)), idempotency of $\sqcap_{0}^{R}$ and functoriality of $\circ$.

Lemma 3.4. For any right weak bimonoid $A$ in a duoidal category $(\mathcal{M}, \circ, \bullet)$ in which idempotent morphisms split, there is a contractible coequalizer of left J-modules

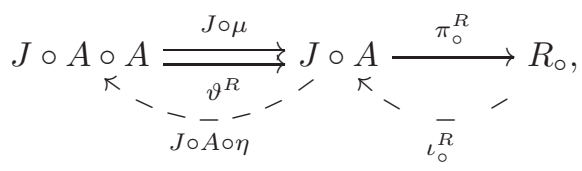

where $\vartheta^{R}$ denotes the morphism

$$
\begin{aligned}
& J \circ A \circ A \stackrel{J \circ A \circ \Delta}{\longrightarrow} J \circ A \circ(A \bullet A) \stackrel{\zeta}{\rightarrow}(J \circ A) \bullet(J \circ A \circ A) \stackrel{(J \circ A) \bullet(J \circ \varepsilon, \mu)}{\longrightarrow}(J \circ A) \bullet(J \circ J) \rightarrow J \circ A \\
= & J \circ A \circ A \stackrel{(J \circ A) \bullet \varpi}{\longrightarrow} J \circ A \circ(A \bullet A) \stackrel{J \circ \zeta}{\rightarrow} J \circ((J \circ A) \bullet(A \circ A)) \stackrel{J \circ((J \circ A) \bullet \varepsilon . \mu)}{\longrightarrow} J \circ J \circ A \stackrel{\varpi \circ A}{\longrightarrow} J \circ A .
\end{aligned}
$$

Proof. The given forms of $\vartheta^{R}$ are equal by (1.9), (1.1), (1.2), the counitality of $\Delta$, naturality of $\zeta$ and functoriality of both monoidal products.

It follows by its form in (3.3) that $\Pi_{0}^{R}$ is a morphism of left $J$-modules. Hence so are $\pi_{\circ}^{R}$ and $\iota_{\circ}^{R}$ with respect to the $J$-action on $R_{\circ}$ in Lemma 3.3 proving that all morphisms in the diagram are 
$J$-linear. By construction, $\iota_{\circ}^{R}$ is a section of $\pi_{\circ}^{R}$ and $J \circ A \circ \eta$ is a section of $J \circ \mu$. Both composites $\iota_{\circ}^{R} \cdot \pi_{\circ}^{R}$ and $\vartheta^{R} .(J \circ A \circ \eta)$ are equal to $\Pi_{\circ}^{R}$ by virtue of (3.2). Finally, in the commutative diagram

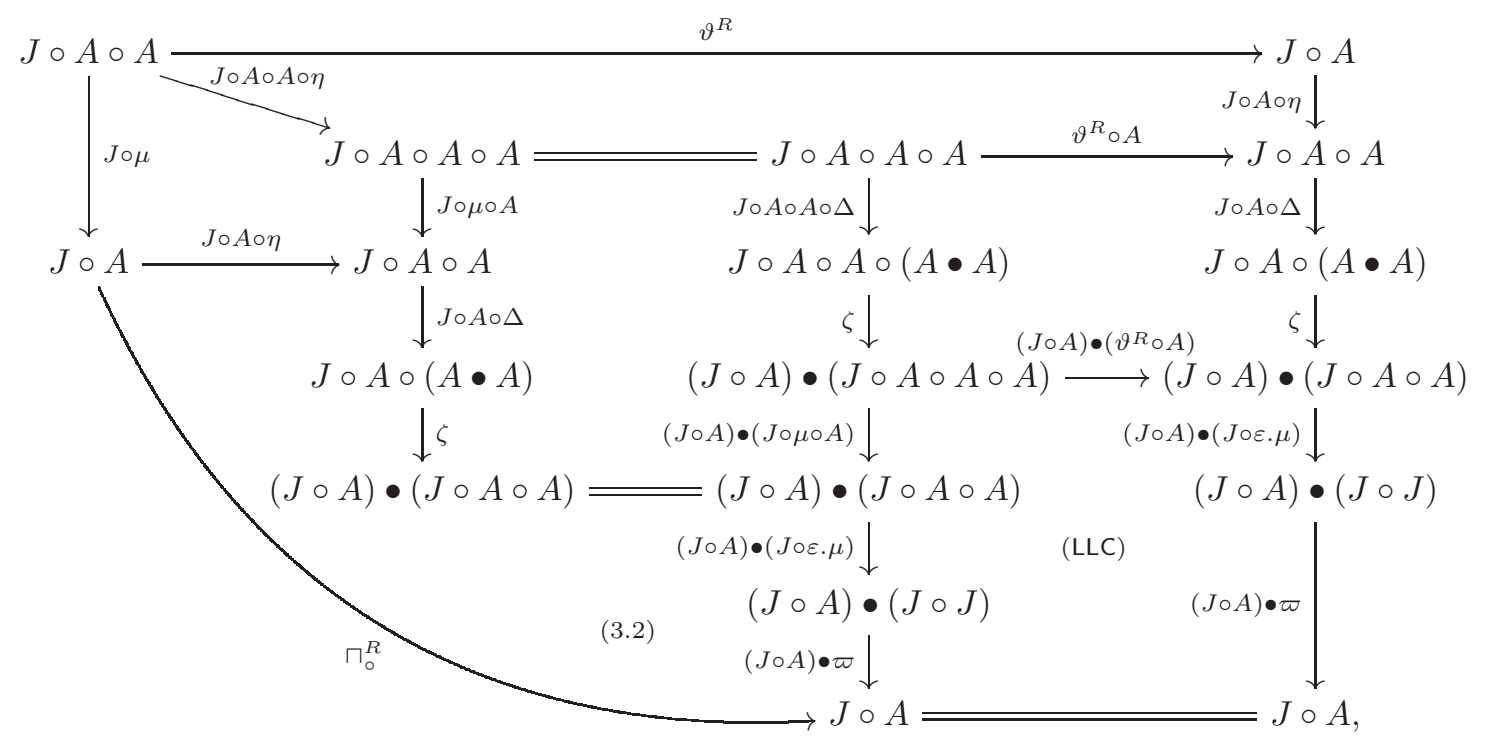

the right vertical is equal to $\sqcap_{0}^{R}=\iota_{\circ}^{R} \cdot \pi_{\circ}^{R}$ by (3.2). Since $\iota_{\circ}^{R}$ is a (split) monomorphism, this proves that the solid arrows in the diagram in the claim constitute a fork.

Lemma 3.5. For any right weak bimonoid $A$ in a duoidal category $(\mathcal{M}, \circ, \bullet)$ in which idempotent morphisms split, $R_{\circ}$ is a separable Frobenius monoid in the category of left $J$-modules.

Proof. We need to show that the structure morphisms (3.4) are left $J$-linear. It is straightforward to check that $J \circ A$ is a comonoid in the category of left $J$-modules, with comultiplication and counit

$$
J \circ A \stackrel{J \circ \Delta}{\longrightarrow} J \circ(A \bullet A) \stackrel{\zeta}{\longrightarrow}(J \circ A) \bullet(J \circ A) \quad \text { and } \quad J \circ A \stackrel{J \circ \varepsilon}{\longrightarrow} J \circ J \stackrel{\varpi}{\longrightarrow} J .
$$

Since $\pi_{\circ}^{R}: J \circ A \rightarrow R_{\circ}$ is a morphism of comonoids, also $R_{\circ}$ is a comonoid in the category of left $J$-modules by Lemma 3.4

It follows by Lemma 3.3 that the left $J$-action on $R_{\circ}$ is a morphism of right $A$-modules. Hence it can be deduced from the naturality of $\chi^{R}$, the naturality and associativity of $\zeta$ and the functoriality of the monoidal product $\circ$, that $\chi_{R_{\circ}, R_{\circ}}^{R}$ is a morphism of left $J$-modules. Since $\chi_{R_{\circ}, R_{\circ}}^{R}=\Delta_{\circ}^{R} \cdot \mu_{\circ}^{R}$ and $\Delta_{\circ}^{R}$ is a morphism of left $J$-modules and also a (split) monomorphism, $\mu_{\circ}^{R}$ is a morphism of $J$-modules too. Finally, the unit $\eta_{\circ}^{R}$ is a morphism of left $J$-modules since $\pi_{\circ}^{R}$ is so by Lemma 3.4

Lemma 3.6. Let $A$ be a right weak bimonoid in a duoidal category $(\mathcal{M}, \circ, \bullet)$ in which idempotent morphisms split. Regard $A$ as a left $R_{\circ}$-comodule via the coaction

$$
\varrho: A \stackrel{\Delta}{\longrightarrow} A \bullet A \stackrel{(\tau \circ A) \bullet A}{\longrightarrow}(J \circ A) \bullet A \stackrel{\pi_{\circ}^{R} \bullet A}{\longrightarrow} R_{\circ} \bullet A
$$


in terms of the comonoid morphism in Lemma 3.2; and regard $A \circ A$ as a left $R_{\circ}$-comodule via the coaction

$$
A \circ A \stackrel{A \circ \varrho}{\longrightarrow} A \circ\left(R_{\circ} \bullet A\right) \stackrel{\zeta}{\longrightarrow}\left(J \circ R_{\circ}\right) \bullet(A \circ A) \stackrel{\gamma \bullet(A \circ A)}{\longrightarrow} R_{\circ} \bullet(A \circ A)
$$

in terms of the action $\gamma: J \circ R_{\circ} \rightarrow R_{\circ}$ (cf. Lemma 3.3). Then the multiplication $\mu: A \circ A \rightarrow A$ is a morphism of left $R_{\circ}$-comodules.

Proof. The coaction on $A \circ A$ is counital and coassociative since $R_{\circ}$ is a comonoid in the category of left $J$-modules (cf. Lemma 3.5) and by the counitality and the coassociativity of $\varrho$, using also an associativity axiom in (1.1), naturality of $\zeta$ and functoriality of the monoidal product $\bullet$. Then the claim follows by commutativity of the diagram in the Appendix, on page 32. In the diagram on page 32, the region marked by (A) commutes by the second explicit form of the morphism $\vartheta^{R}$ in Lemma 3.4, unitality of $\varpi$ and by functoriality of the monoidal product $\bullet$. The region marked by (B) commutes since $\pi_{\circ}^{R}$ coequalizes the parallel morphisms in Lemma 3.4. The region marked by (C) commutes since $\pi_{\circ}^{R}$ is a morphism of left $J$-modules by Lemma 3.4

Symmetric considerations apply to the Eilenberg-Moore categories of all weak bi(co)monads in Corollary 2.6.

\section{THE BASE OBJECTS}

Let $A$ be a weak bimonoid in a duoidal category $(\mathcal{M}, \circ, \bullet)$ in which idempotent morphisms split. Applying the transformations $\circ, \bullet$ and $*$ in Section 2, and all of their composites to the morphism $\Pi_{0}^{R}$ in (3.2), we obtain an eight member family of idempotent morphisms fitting the diagram

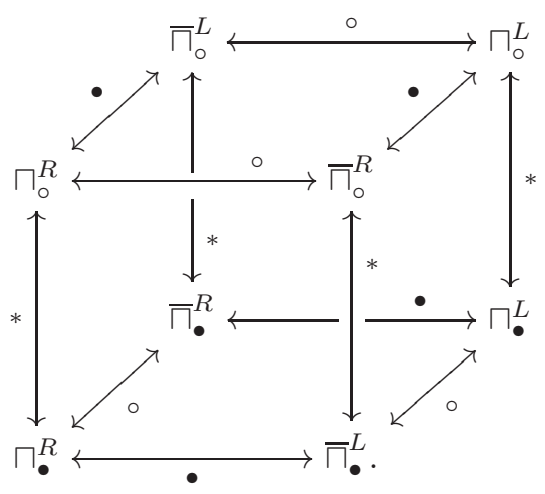

Their splittings define the respective objects $\bar{L}_{\circ}, L_{\circ}, R_{\circ}, \bar{R}_{\circ}, \bar{R}_{\bullet}, L_{\bullet}, R_{\bullet}$ and $\bar{L}_{\bullet}$ in $\mathcal{M}$. Each of them carries a separable Frobenius monoid structure in the appropriate monoidal category $(\mathcal{M}, \circ)$ or $(\mathcal{M}, \bullet)$. Symmetric considerations to those in Section 3 apply to them. For example, by symmetric versions of Lemma 3.2 , there are comonoid morphisms from $A$ to $\bar{L}_{\circ}, L_{\circ}, R_{\circ}$ and $\bar{R}_{\circ}$; and there are monoid morphisms from $\bar{R}_{\bullet}, L_{\bullet}, R_{\bullet}$ and $\bar{L}_{\bullet}$ to $A$.

If $A$ is a weak bimonoid in a braided monoidal category - regarded as a duoidal category — whose idempotent morphisms split, then $R_{\circ}, R_{\bullet}$ and $\bar{R}_{\bullet}$ become isomorphic to the 'right' or 'source' Frobenius monoid, while $\bar{R}_{\circ}$ becomes isomorphic to the opposite monoid and opposite comonoid. Symmetrically, $L_{\circ}, L_{\bullet}$ and $\bar{L}_{\bullet}$ become isomorphic to the 'left' or 'target' Frobenius monoid, while $\bar{L}_{\circ}$ becomes isomorphic to the opposite monoid and opposite comonoid. Thus all 
the eight base Frobenius monoids become (anti-)isomorphic (cf. 22]). The aim of this section is to relate these objects (that we call the 'base objects' of $A$ ) in our more general setting.

Applying the isomorphism in (1.8) to the weak bi(co)monads in (2.2), we obtain the following.

Proposition 4.1. For any weak bimonoid $A$ in a duoidal category $(\mathcal{M}, \circ, \bullet)$ in which idempotent morphisms split, the following objects of $\mathcal{M}$ (defined above) are pairwise isomorphic.

(1) $L_{\bullet} \cong \bar{L}_{\bullet}$ as monoids and comonoids in $(\mathcal{M}, \circ)$.

(2) $R_{\bullet} \cong \bar{R}_{\bullet}$ as monoids and comonoids in $(\mathcal{M}, \circ)$.

(3) $L_{\circ} \cong \bar{R}_{\circ}$ as monoids and comonoids in $(\mathcal{M}, \bullet)$.

(4) $R_{\circ} \cong \bar{L}_{\circ}$ as monoids and comonoids in $(\mathcal{M}, \bullet)$.

Note that by splitting certain idempotent morphisms, the objects occurring in Proposition 4.1 are defined only up-to isomorphism. Hence without any loss of generality, we may identify the isomorphic objects in parts (1)-(4). We will do so throughout the paper, replacing the objects written on the right hand side in parts (1)-(4) of Proposition 4.1 with their isomorphic copies on the left hand side.

Proposition 4.2. For any weak bimonoid $A$ in a duoidal category $(\mathcal{M}, \circ, \bullet)$ in which idempotent morphisms split, the following objects of $\mathcal{M}$ (defined above) are pairwise isomorphic.

(1) $L_{\circ} \cong L_{\bullet} \circ J$ as $L_{\bullet}-J$ bimodules.

(2) $L_{\circ} \cong R_{\bullet} \circ J$ as $R_{\bullet}-J$ bimodules.

(3) $R_{\circ} \cong J \circ R_{\bullet}$ as $J-R \bullet$ bimodules.

(4) $R_{\circ} \cong J \circ L$. as $J-L$ • bimodules.

(5) $L_{\bullet} \cong L_{\circ} \bullet I$ as $L_{\circ}-I$ bicomodules.

(6) $L_{\bullet} \cong R_{\circ} \bullet I$ as $R_{\circ}-I$ bicomodules.

(7) $R_{\bullet} \cong I \bullet R_{\circ}$ as $I-R_{\circ}$ bicomodules.

(8) $R_{\bullet} \cong I \bullet L_{\circ}$ as $I-L_{\circ}$ bicomodules.

Proof. We only prove part (1), all other claims follow from it applying the transformations $\circ, \bullet, *$ and their composites. Consider the morphisms $\phi:(A \bullet I) \circ J \stackrel{(A \bullet \tau) \circ J}{\longrightarrow} A \circ J$ and

$$
\begin{aligned}
\phi^{\prime}: A \circ J \stackrel{\delta \circ A \circ J}{\longrightarrow}(I \bullet I) \circ A \circ J \stackrel{(\Delta . \eta \bullet I) \circ A \circ J}{\longrightarrow}(A \bullet A \bullet I) \circ A \circ J \stackrel{\zeta}{\longrightarrow} \\
(A \circ A \circ J) \bullet((A \bullet I) \circ J) \stackrel{(\varepsilon . \mu \circ J) \bullet((A \bullet I) \circ J)}{\longrightarrow}(J \circ J) \bullet((A \bullet I) \circ J) \longrightarrow(A \bullet I) \circ J .
\end{aligned}
$$


By functoriality of both monoidal products, naturality of $\zeta$ and counitality of $\delta$, it follows that $\phi . \phi^{\prime}=\sqcap_{\circ}^{L}$. On the other hand, by commutativity of

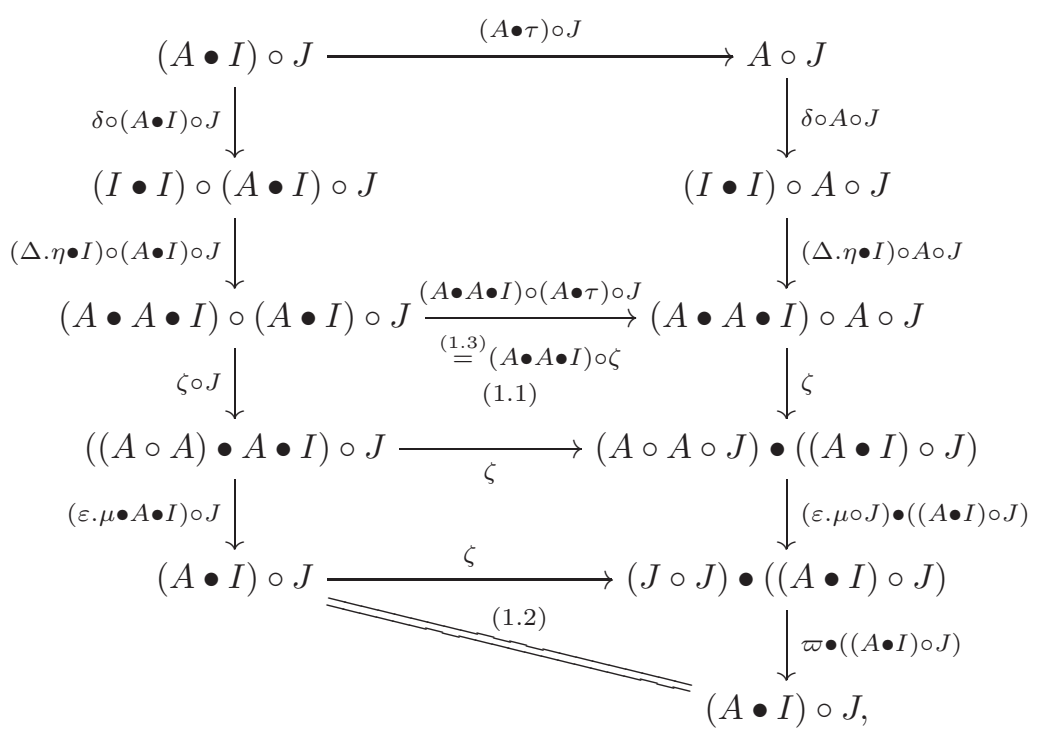

also $\phi^{\prime} . \phi=\sqcap_{\bullet}^{L} \circ J$. Hence $\phi$ and $\phi^{\prime}$ (co)restrict to the stated isomorphisms

$$
L_{\bullet} \circ J \stackrel{\iota_{\bullet}^{L} \circ J}{\rightarrow}(A \bullet I) \circ J \stackrel{\phi}{\rightarrow} A \circ J \stackrel{\pi_{\circ}^{L}}{\rightarrow} L_{\circ} \quad \text { and } \quad L_{\circ} \stackrel{\iota_{\circ}^{L}}{\rightarrow} A \circ J \stackrel{\phi^{\prime}}{\rightarrow}(A \bullet I) \circ J \stackrel{\pi_{\bullet}^{L} \circ J}{\rightarrow} L \bullet \circ J .
$$

By a symmetric version of Lemma 3.3, $L_{\circ}$ is an $A-J$ bimodule. Hence it is an $L_{\bullet}-J$ bimodule via restriction along the monoid morphism $L \bullet \stackrel{\iota_{\bullet}^{L}}{\longrightarrow} A \bullet I \stackrel{A \bullet \tau}{\longrightarrow} A$ (cf. a symmetric form of Lemma 3.2) . The morphisms $\phi$ and $\Pi_{\bullet}^{L} \circ J$ are evidently right $J$-module maps and so is $\Pi_{\circ}^{L}$ by a symmetric version of Lemma 3.4. Hence the induced isomorphism $L_{\bullet} \circ J \rightarrow L_{\circ}$ is also a morphism of right $J$-modules. Since $L_{\bullet} \circ \pi_{\bullet}^{L} \circ J$ is a (split) epimorphism, the isomorphism $L_{\bullet} \circ J \rightarrow L_{\circ}$ is also a morphism of left $L_{\bullet}$-modules by commutativity of

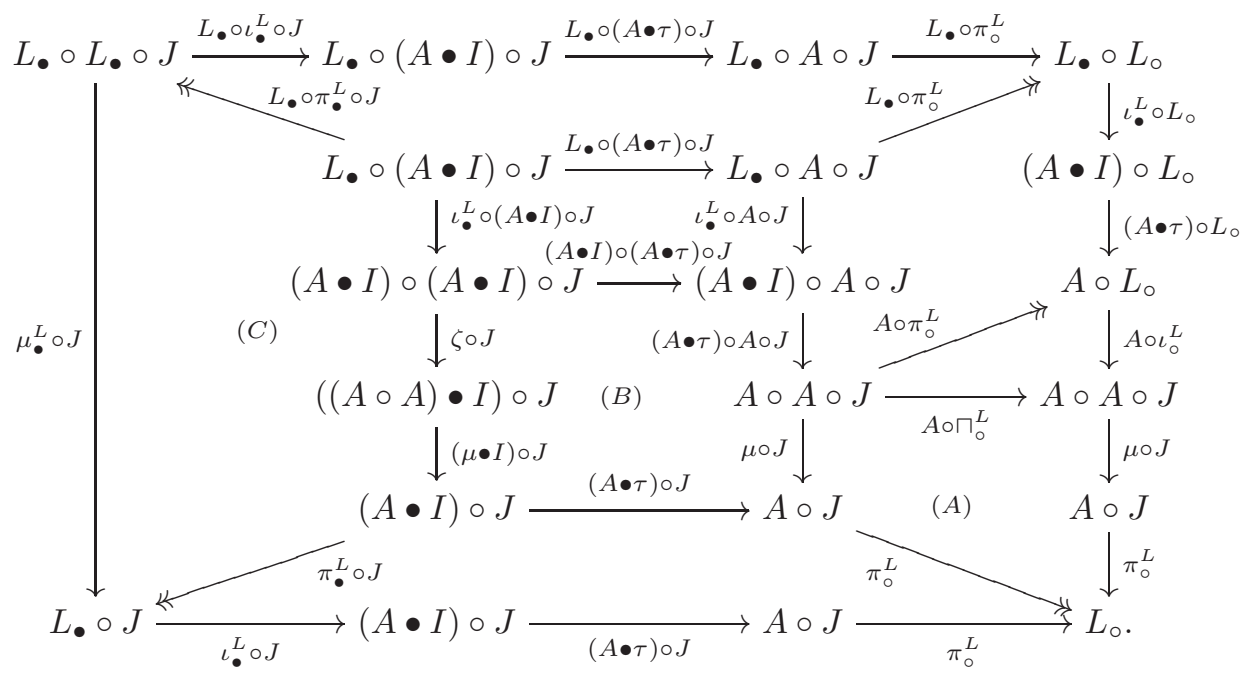

The region marked by $(A)$ commutes by (1.7). The region marked by $(B)$ commutes since $A \bullet \tau$ : $A \bullet I \rightarrow A$ is a monoid morphism (by a dual version of the reasoning in the proof of Lemma 3.2. ). 
The region marked by $(C)$ commutes since $\pi_{\bullet}^{L}$ is a morphism of left $L_{\bullet}$-modules by a symmetric version of Lemma 3.1

\section{Hopf modules}

Hopf modules over a (weak) bialgebra are both modules and comodules with an appropriate compatibility condition between the action and the coaction. The compatibility condition is most conveniently formulated in terms of a '(weak) mixed distributive law' (called a '(weak) entwining structure' in [14]). Our definition of Hopf modules over weak bimonoids in a duoidal category in this section, uses this language.

Proposition 5.1. For any weak bimonoid $A$ in a duoidal category $(\mathcal{M}, \circ, \bullet)$, there is a weak mixed distributive law between the induced monad $(-) \circ A$ and comonad $(-) \bullet A$ on $\mathcal{M}$.

Proof. We construct the desired weak mixed distributive law putting

$$
\psi_{M}:(M \bullet A) \circ A \stackrel{(M \bullet A) \circ \Delta}{\longrightarrow}(M \bullet A) \circ(A \bullet A) \stackrel{\zeta}{\longrightarrow}(M \circ A) \bullet(A \circ A) \stackrel{(M \circ A) \bullet \mu}{\longrightarrow}(M \circ A) \bullet A,
$$

for any object $M \in \mathcal{M}$. The compatibility

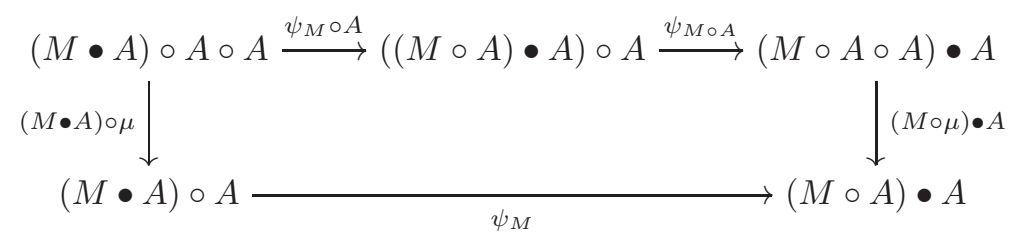

with the multiplication follows from the first axiom (2.1) of weak bimonoids, the associativity axioms (1.1) of a duoidal category and associativity of the monoid $A$, together with the naturality of $\zeta$ and functoriality of both monoidal products. (This condition is proven in the same way as in the non-weak case). The compatibility

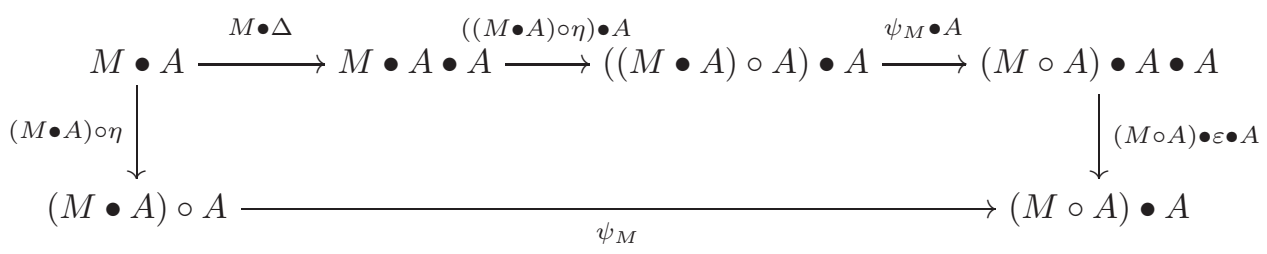

with the unit is proven in the Appendix on page 33. The compatibility conditions with the comultiplication and the counit are obtained by applying the transformation $*$ to the above diagrams. Hence they follow by symmetry.

Applying the duality transformations $\bullet$, o and their composite to the weak distributive law $\psi$ in Proposition 5.1, we obtain a four member family of weak distributive laws between the various induced (co)monads. In the rest of the paper we always work with $\psi$ in Proposition 5.1 but certainly symmetric considerations apply to all of its dual counterparts.

Over the weak mixed distributive law $\psi$ in Proposition 5.1 , we may consider the mixed modules [6, 9] in the following sense. 
Definition 5.2. Mixed modules over the weak mixed distributive law $\psi$ in Proposition 5.1 are called Hopf modules over the weak bimonoid $A$. Explicitly, this means an object $M$ in $\mathcal{M}$ which is both a $(-) \circ A$-module with action $\gamma$ and a $(-) \bullet A$-comodule with coaction $\varrho$ such that the following diagram commutes.

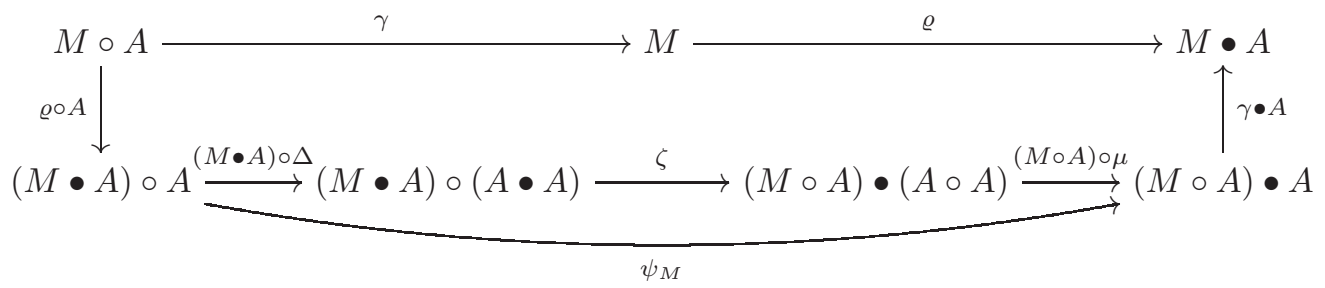

Morphisms of Hopf modules are morphisms of $(-) \bullet A$-comodules and $(-) \circ A$-modules. The category of Hopf modules is denoted by $\mathcal{M}_{A}^{A}$.

By the weak bimonoid axiom (2.1), $A$ is a Hopf module over itself via the multiplication and the comultiplication.

For any monad $t$ and comonad $c$ on the same category $\mathcal{M}$, it follows by [23, Corollary 5.11 and Proposition 6.3] that (non-weak) mixed distributive laws $t c \rightarrow c t$ are in a bijective correspondence with liftings $\bar{c}$ of the comonad $c$ to the Eilenberg-Moore category $\mathcal{M}^{t}$ of the monad $t$. This means that the functor $\bar{c}$ renders commutative the diagram

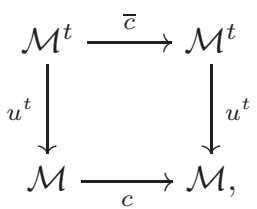

involving the forgetful functor $u^{t}$. Moreover, the comultiplication $\bar{\delta}$ and the counit $\bar{\varepsilon}$ of the comonad $\bar{c}$ are related to the comultiplication $\delta$ and the counit $\varepsilon$ of $c$ via $u^{t} \bar{\delta}=\delta u^{t}$ and $u^{t} \bar{\varepsilon}=\varepsilon u^{t}$.

In a similar way, by [6. Proposition 5.7], whenever idempotent morphisms in $\mathcal{M}$ split, a weak mixed distributive law $t c \rightarrow c t$ determines a weak lifting $\bar{c}$ of $c$ to $\mathcal{M}^{t}$. This means that the diagram in (5.2) does not need strictly commute. Instead, there are natural transformations $\iota: u^{t} \bar{c} \rightarrow c u^{t}$ and $\pi: c u^{t} \rightarrow u^{t} \bar{c}$ such that their composite $\pi . \iota$ is the identity (they are obtained by splitting an idempotent natural transformation canonically associated to the weak mixed distributive law). Moreover, $\left(u^{t}, \iota\right)$ is a comonad morphism from $\bar{c}$ to $c$ in the sense of [26].

In the situation of Proposition 5.1 this means the following. Associated to the weak mixed distributive law $\psi$ in Proposition 5.1 for any $(-) \circ A$-module $(Q, \gamma)$ there is an idempotent morphism

$$
Q \bullet A \stackrel{(Q \bullet A) \circ \eta}{\longrightarrow}(Q \bullet A) \circ A \stackrel{\psi_{Q}}{\longrightarrow}(Q \circ A) \bullet A \stackrel{\gamma \bullet A}{\longrightarrow} Q \bullet A .
$$

In light of the explicit form of $\psi_{Q}$, it is equal to $\chi_{Q, A}^{R}$ in (3.1). So whenever idempotent morphisms in $\mathcal{M}$ split, it splits through the $R_{\circ}$-module product $Q \bullet_{R_{\circ}} A$. This defines a comonad $(-) \bullet_{R_{\circ}} A$ on the category $\mathcal{M}_{A}$ of $(-) \circ A$-modules, which is the weak lifting of the comonad $(-) \bullet A$ on $\mathcal{M}$, induced by the weak mixed distributive law $\psi$ in Proposition 5.1 via the correspondence in 6 , Proposition 5.7]. As proven in [6, Proposition 3.7], the Eilenberg-Moore category of comodules over this weakly lifted comonad $(-) \bullet_{R_{\circ}} A$ on $\mathcal{M}_{A}$, is isomorphic to the category $\mathcal{M}_{A}^{A}$ of mixed modules 
over $\psi$. By these considerations, whenever idempotent morphisms in $\mathcal{M}$ split, the forgetful functor $\mathcal{M}_{A}^{A} \rightarrow \mathcal{M}_{A}$ is comonadic.

Proposition 5.3. Let $A$ be a weak bimonoid in a duoidal category $(\mathcal{M}, \circ, \bullet)$. Then the functor $(-) \circ A: \mathcal{M} \rightarrow \mathcal{M}_{A}$ lifts to $\mathcal{M}^{I} \rightarrow \mathcal{M}_{A}^{A}$ (where I stands for the o-monoidal unit regarded as a comonoid in $(\mathcal{M}, \bullet)$, and $\mathcal{M}^{I}$ denotes the category of $(-) \bullet$ I-comodules $)$. That is, there is a functor $\mathcal{M}^{I} \rightarrow \mathcal{M}_{A}^{A}$ rendering commutative the diagram

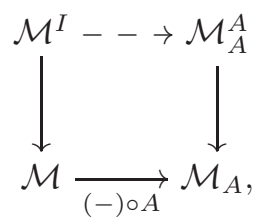

in which the vertical arrows denote the forgetful functors.

Proof. The object map of the desired functor is provided by the action and the coaction

$$
Z \circ A \circ A \stackrel{Z \circ \mu}{\longrightarrow} Z \circ A \quad Z \circ A \stackrel{\varrho \circ \Delta}{\longrightarrow}(Z \bullet I) \circ(A \bullet A) \stackrel{\zeta}{\longrightarrow}(Z \circ A) \bullet A,
$$

for any $(-) \bullet I$-comodule $(Z, \varrho)$. The action is associative and unital by the associativity and unitality of the multiplication. The coaction is coassociative by coassociativity of $\Delta$ and $\varrho$ and the axioms (1.1) and (1.2), together with the naturality of $\zeta$. It is counital by (1.3), the counitality of $\Delta$ and $\varrho$, and the naturality of $\zeta$. The compatibility condition (5.1) follows by the weak bimonoid axiom (2.1), an associativity axiom in (1.1), and the naturality of $\zeta$ and functoriality of the monoidal product $\circ$. Obviously, for any $(-) \bullet I$-comodule morphism $f, f \circ A$ becomes a morphism of Hopf modules with respect to these actions and coactions.

Whenever idempotent morphisms in $\mathcal{M}$ split - hence $\mathcal{M}_{A}^{A}$ is isomorphic to the EilenbergMoore category of the comonad $(-) \bullet_{R_{\circ}} A$ on $\mathcal{M}_{A}$ - it follows by [23, Corollary 5.11] that the lifted functor in Proposition 5.3 corresponds to a comonad morphism (in the sense of [26]), $\lambda^{0}:((-) \bullet I) \circ A \rightarrow((-) \circ A) \bullet_{R_{\circ}} A$, between functors $M \rightarrow M_{A}$. Applying a dual form of [6] Lemma 3.6] — yielding the explicit correspondence between the $A$-Hopf modules and the comodules of the comonad $(-) \bullet_{R_{\circ}} A$ on $\mathcal{M}_{A}$-, for any object $M$ of $\mathcal{M}$ the explicit form of $\lambda_{M}^{0}$ comes out as

$$
\lambda_{M}^{0}:(M \bullet I) \circ A \stackrel{(M \bullet I) \circ \Delta}{\longrightarrow}(M \bullet I) \circ(A \bullet A) \stackrel{\zeta}{\longrightarrow}(M \circ A) \bullet A \stackrel{\pi_{M \circ A, A}^{R}}{\longrightarrow}(M \circ A) \bullet_{R_{\circ}} A .
$$

In other words, $\lambda_{M}^{0}$ is the unique morphism for which

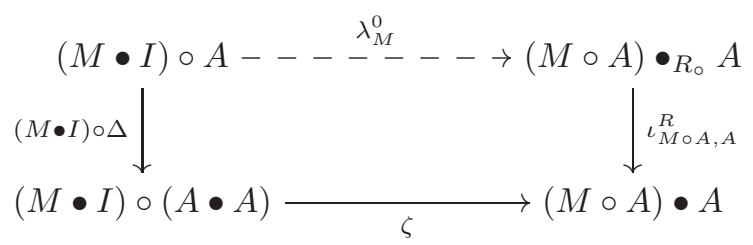


commutes. Both definitions of $\lambda_{M}^{0}$ are equivalent since

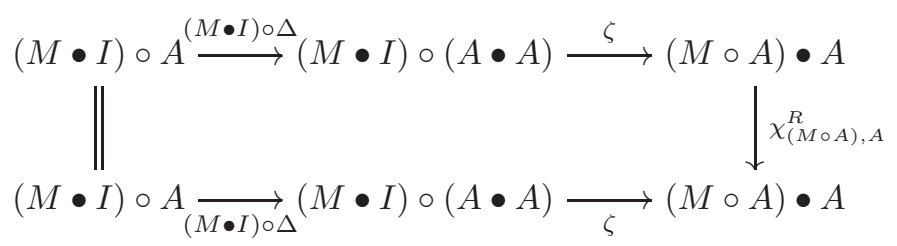

commutes by (1.1), (2.1) and unitality of the monoid $A$, together with the naturality of $\zeta$ and functoriality of o. As in [15, Proposition 1.1], the comonad morphism $\lambda^{0}$ determines a ('Galoistype') comonad morphism

$$
\beta_{Q}^{0}:(Q \bullet I) \circ A \stackrel{\lambda_{Q}^{0}}{\longrightarrow}(Q \circ A) \bullet_{R_{\circ}} A \stackrel{\gamma_{R_{\circ}} A}{\longrightarrow} Q \bullet_{R_{\circ}} A,
$$

for any right $A$-module $(Q, \gamma)$. It is in fact the unique morphism for which

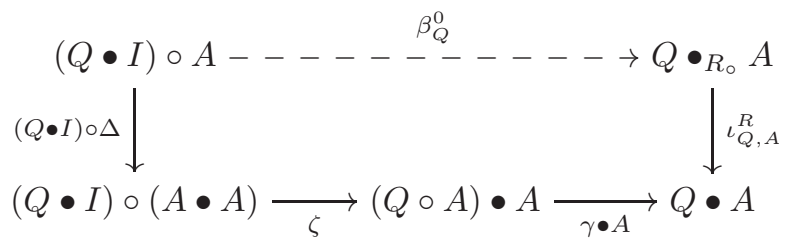

commutes.

Regard the symmetric monoidal category of vector spaces as a duoidal category. Clearly, its idempotent morphisms split. A weak bimonoid $A$ in this duoidal category is the usual notion of weak bialgebra in [11, 20, 10]. By [13, Section 36.16], the fundamental theorem of Hopf modules holds for $A$ if and only if it is a weak Hopf algebra. In this case, the fundamental theorem asserts that a certain comparison functor, from the category of modules over the 'left' or 'target' algebra to the category of $A$-Hopf modules, is an equivalence. In order to obtain the generalization of this comparison functor in our setting of duoidal categories, it is not enough to consider the lifted functor in Proposition [5.3, we need another lifting.

If $A$ is a weak bimonoid in a duoidal category $(\mathcal{M}, \circ, \bullet)$ in which idempotent morphisms split, then it follows by a symmetric version of Lemma 3.4 that $\bar{L}$ • (and hence by Proposition 4.1 (1) also the isomorphic object $L$ • with which we identified it) fits the equalizer

$$
L \bullet \stackrel{\iota_{\bullet}^{L}}{\longrightarrow} A \bullet I \frac{\Delta \bullet I}{\overline{\vartheta^{L}:=(\mu \bullet A \bullet I) .(\zeta \bullet I) .(((A \bullet I) \circ \Delta . \eta) \bullet I) .(A \bullet \delta)}} A \bullet A \bullet I
$$

in $\mathcal{M}^{I}$.

We know from (a symmetric counterpart of) Lemma 3.5 that $L_{\bullet}$ is a separable Frobenius monoid in $\mathcal{M}^{I}$. In particular, $L_{\bullet}$ is a monoid in $\mathcal{M}^{I}$, so we can take its category of modules $\mathcal{M}_{L_{\bullet}}^{I}$. Equivalently, $\mathcal{M}_{L \bullet}^{I}$ is the category of comodules over the comonad (-) $\bullet I$ on $\mathcal{M}_{L_{\bullet}}$. Thus it comes equipped with a forgetful functor $V^{I}: \mathcal{M}_{L_{\bullet}}^{I} \rightarrow \mathcal{M}_{L_{\bullet}}$, with right adjoint $(-) \bullet I$.

By a symmetric version of Lemma 3.2 there is a monoid morphism $\omega: L \bullet \stackrel{\iota^{L}}{\rightarrow} A \bullet I \stackrel{A \bullet \tau}{\rightarrow} A$ in $(\mathcal{M}, \circ)$. It induces a left $L_{\bullet}$-action on any left $A$-module and hence it induces in particular a left 
$L_{\bullet}$-action $\alpha$ on $A$. For any right $L_{\bullet}$-module $(P, \xi)$, the $L_{\bullet}$-module tensor product $P \circ_{L_{\bullet}} A$ is defined - if it exists - as the coequalizer

$$
P \circ L_{\bullet} \circ A \underset{P \circ \alpha}{\stackrel{\xi \circ A}{\longrightarrow}} P \circ A \stackrel{\pi_{P, A}^{L}}{\longrightarrow} P \circ_{L} . A
$$

in $\mathcal{M}$. Recall (e.g. from [22, Section 3.1]) that, since $L_{\bullet}$ is a separable Frobenius monoid (in $(\mathcal{M}, \circ)), P \circ_{L} . A$ can be obtained also by splitting the idempotent morphism

$$
P \circ A \stackrel{P \circ \Delta_{\bullet}^{L} \cdot \eta_{\bullet}^{L} \circ A}{\longrightarrow} P \circ L_{\bullet} \circ L_{\bullet} \circ A \stackrel{\xi \circ \alpha}{\longrightarrow} P \circ A
$$

as $P \circ A-\pi_{P, A}^{L} \rightarrow P \circ_{L}, A \succ \iota_{P, A}^{L} \rightarrow P \circ A$. Hence by the assumption that idempotent morphisms in $\mathcal{M}$ split, the $L_{\bullet}$-module tensor product $P \circ_{L_{\bullet}} A$ exists, and it is preserved by any functor. This defines a functor $\omega^{*}:=(-) \circ_{L}, A: \mathcal{M}_{L_{\bullet}} \rightarrow \mathcal{M}_{A}$. It is left adjoint of the functor $\omega_{*}: \mathcal{M}_{A} \rightarrow \mathcal{M}_{L}$. defined by regarding any $A$ module as an $L_{\bullet}$-module via $\omega$ (see e.g. [21]).

Lemma 5.4. Let $A$ be a weak bimonoid in a duoidal category $(\mathcal{M}, \circ, \bullet)$ whose idempotent morphisms split. Then for any right $L_{\bullet}$-module $(P, \xi)$,

$$
(P \bullet I) \circ L \bullet \circ A \underset{(P \bullet I) \circ \alpha}{\stackrel{\gamma \circ A}{\longrightarrow}}(P \bullet I) \circ A \stackrel{\left(\pi_{P, A}^{L} \bullet_{R_{\circ}} A\right) \cdot \lambda_{P}^{0}}{\longrightarrow}\left(P \circ_{L} \cdot A\right) \bullet R_{\circ} A
$$

is a fork, where $\lambda^{0}$ is the comonad morphism in (5.3), $\alpha=\mu .(\omega \circ A)$ is the L-action on $A$ induced by the monoid morphism $\omega=(A \bullet \tau) . \iota_{\bullet}^{L}: L_{\bullet} \rightarrow A$ and $\gamma$ is the $L_{\bullet}$-action on $P \bullet I$, given in terms of the I-coaction $\varrho: L_{\bullet} \rightarrow L_{\bullet} \bullet I$ from a dual form of Lemma 3.3 as

$$
(P \bullet I) \circ L \bullet \stackrel{(P \bullet I) \circ \varrho}{\longrightarrow}(P \bullet I) \circ(L \bullet \bullet I) \stackrel{\zeta}{\longrightarrow}(P \circ L \bullet) \bullet I \stackrel{\xi \bullet I}{\longrightarrow} P \bullet I .
$$

Proof. The proof can be found in the Appendix, on page 34. In the diagram on page 34, the region marked by 'Lemma 3.6' commutes since by a symmetric version of Lemma 3.6, $\Delta: A \rightarrow A \bullet A$ is a morphism of left $\bar{L} \bullet L_{\bullet}$-modules (for the isomorphism see Proposition 4.1).

Proposition 5.5. Let $A$ be a weak bimonoid in a duoidal category $(\mathcal{M}, \circ, \bullet)$ in which idempotent morphisms split. Then the functor $\omega^{*}=(-){ }^{\circ} L_{\bullet} A: \mathcal{M}_{L_{\bullet}} \rightarrow \mathcal{M}_{A}$ above lifts to $\mathcal{M}_{L_{\bullet}}^{I} \rightarrow \mathcal{M}_{A}^{A}$. That is, there is a functor $\mathcal{M}_{L}^{I} \rightarrow \mathcal{M}_{A}^{A}$, rendering commutative the diagram

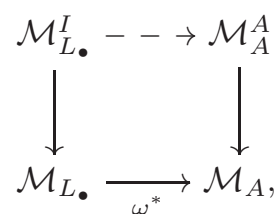

in which the vertical arrows denote the (comonadic) forgetful functors.

Proof. The category $\mathcal{M}_{A}^{A}$ of Hopf modules is isomorphic to the Eilenberg-Moore category of comodules over the comonad $(-) \bullet_{R_{0}} A$ on $\mathcal{M}_{A}$. Hence using the bijection between liftings and comonad morphisms (see e.g. [23, Corollary 5.11]), the proof amounts to constructing a morphism $\lambda_{P}:(P \bullet I) \circ_{L_{\bullet}} A \rightarrow\left(P \circ_{L}, A\right) \bullet_{R_{\circ}} A$ in $\mathcal{M}_{A}$, for any right $L_{\bullet}$-module $(P, \xi)$; and showing that 
$\lambda$ is in fact a comonad morphism (in the sense of [26]). Using the notation in Lemma [5.4, in the diagram

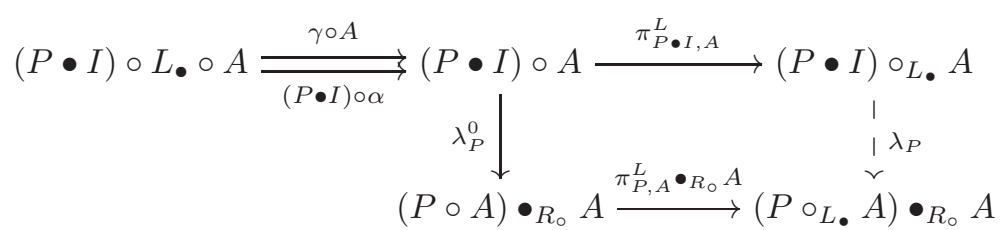

the top row is a coequalizer as in (5.5). By Lemma 5.4 $\left(\pi_{P, A}^{L} \bullet_{R_{\circ}} A\right) \cdot \lambda_{P}^{0}$ coequalizes the parallel morphisms in the top row. So by universality, the (unique) morphism $\lambda_{P}$ exists. Since $\lambda^{0}$ and $\pi^{L}$ are natural, so is $\lambda$ by its construction in (5.8). We know from Proposition 5.3 that $\lambda_{P}^{0}$ is a morphism of (-)॰A-modules, hence so is $\left(\pi_{P, A}^{L} \bullet R_{\circ} A\right) . \lambda_{P}^{0}$. Since (5.6) is a morphism of $(-) \circ A$ modules (with respect to the action given by multiplication in the last factor), so is $\pi_{P, A}^{L}$ for any $L_{\bullet}$-module $P$. From these and (5.8) we conclude that $\lambda_{P}$ is a morphism of $(-) \circ A$-modules. The compatibilities of $\lambda$ with the comultiplications and the counits of the comonads (-) $\bullet$ on $\mathcal{M}_{L}$. and $(-) \bullet_{R_{\circ}} A$ on $\mathcal{M}_{A}$ follow by naturality of $\pi^{L}$ in its first argument, functoriality of the product $\bullet_{R_{\circ}}$ and the compatibilities of $\lambda^{0}$, which are consequences of Proposition 5.3 ,

Corresponding (as in [15, Proposition 1.1]) to the comonad morphism $\lambda$ in Proposition[5.5, there is a 'Galois-type' morphism of comonads

$$
\beta_{Q}:(Q \bullet I) \circ_{L} A \stackrel{\lambda_{Q}}{\longrightarrow}\left(Q \circ_{L} A\right) \bullet_{R_{\circ}} A \stackrel{\bar{\gamma}_{R_{\circ}} A}{\longrightarrow} Q \bullet_{R_{\circ}} A,
$$

for any right $A$-module $(Q, \gamma)$, where $\bar{\gamma}$ is the unique morphism for which $\bar{\gamma} \cdot \pi_{Q, A}^{L}=\gamma$. This is the unique morphism rendering commutative

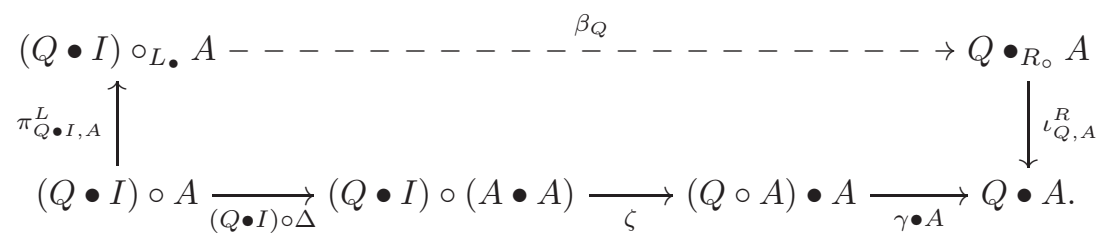

\section{The Fundamental Theorem of Hopf modules}

By Proposition 5.5, there is a functor $(-){ }^{\circ} L_{\bullet} A: \mathcal{M}_{L_{\bullet}}^{I} \rightarrow \mathcal{M}_{A}^{A}$, for any weak bimonoid $A$ in a duoidal category $(\mathcal{M}, \circ, \bullet)$ in which idempotent morphisms split. The aim of this section is to investigate when this is an equivalence; that is, when the fundamental theorem of Hopf modules holds. In the case of a weak bialgebra $A$ over a field, it is known to be the case if and only if $A$ is a weak Hopf algebra (see [13, Section 36.16]).

Let $(\mathcal{M}, \circ, \bullet)$ be a duoidal category in which idempotent morphisms split and let $A$ be a bimonoid in it. In the diagram

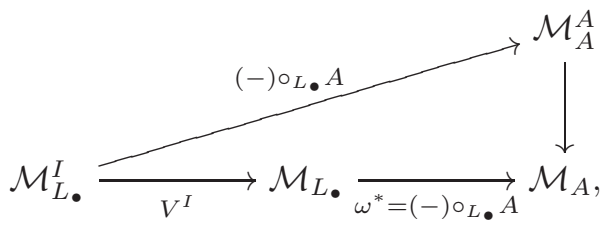


the vertical arrow denotes the comonadic forgetful functor and the functor in the bottom row to be denoted by $Y$ - is left adjoint. Hence the diagonal functor $(-){ }^{\circ}{ }_{L}, A: \mathcal{M}_{L_{\bullet}}^{I} \rightarrow \mathcal{M}_{A}^{A}$ is an equivalence if and only if (5.9) is a natural isomorphism and $Y=(-){ }^{\circ} L_{\bullet} A: \mathcal{M}_{L}^{I} \rightarrow \mathcal{M}_{A}$ is comonadic (see e.g. [15, Theorem 2.7] or [17, Theorem 1.10]). By this motivation, applying methods in [17, we turn to studying when $Y$ is comonadic.

Regard the Kleisli category $\widetilde{\mathcal{M}}_{A}$ of the monad $\omega_{*} \omega^{*}=(-){ }^{\circ} L_{\bullet} A$ on $\mathcal{M}_{L_{\bullet}}$ as a full subcategory (of free modules) in the Eilenberg-Moore category of $\omega_{*} \omega^{*}$; which is isomorphic to $\mathcal{M}_{A}$. The functor $Y=(-) \circ_{L_{\bullet}} A: \mathcal{M}_{L_{\bullet}}^{I} \rightarrow \mathcal{M}_{A}$ factorizes through $\widetilde{Y}:=(-) \circ_{L_{\bullet}} A: \mathcal{M}_{L_{\bullet}}^{I} \rightarrow \widetilde{\mathcal{M}}_{A}$ via the fully faithful embedding $\widetilde{\mathcal{M}}_{A} \rightarrow \mathcal{M}_{A}$.

By a symmetric version of Lemma 3.3. $L_{\circ}$ carries the structure of an $A-J$ bimodule in $(\mathcal{M}, \circ)$. For any object $P \circ_{L} . A$ of $\widetilde{\mathcal{M}}_{A}$ and any left $A$-module $Q$, there exists the coequalizer $P \circ_{L} \cdot A \circ_{A} Q \cong$ $P \circ_{L_{\bullet}} Q$, see e.g. [17, Remark 2.4] (where $Q$ is a left $L_{\bullet}$-module via the action induced by the monoid morphism $\omega=(A \bullet \tau) . \iota_{\bullet}^{L}: L_{\bullet} \rightarrow A$, cf. a symmetric version of Lemma 3.2). Thus for any right $L_{\bullet}$-module $P$, there is a right $J$-module $P \circ_{L_{\bullet}} A \circ_{A} L_{\circ} \cong P \circ_{L_{\bullet}} L_{\circ}$. By Proposition 4.2 , $L_{\circ} \cong L_{\bullet} \circ J$ as $L_{\bullet}-J$ bimodules. Therefore

$$
P \circ_{L_{\bullet}} A \circ_{A} L_{\circ} \cong P \circ_{L_{\bullet}} L_{\circ} \cong P \circ_{L_{\bullet}} L_{\bullet} \circ J \cong P \circ J,
$$

resulting in a commutative (up-to natural isomorphism) diagram

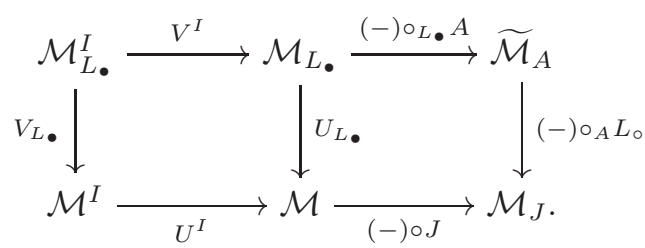

Proposition 6.1. Let $(\mathcal{M}, \circ, \bullet)$ be a duoidal category in which idempotent morphisms split and the functor $\mathcal{M}^{I} \stackrel{U^{I}}{\longrightarrow} \mathcal{M} \stackrel{(-) \circ J}{\longrightarrow} \mathcal{M}_{J}$ in the bottom row of (6.1) is separable (in the sense of [19]). Then for any weak bimonoid $A$ in $\mathcal{M}$, the functor $\widetilde{Y}=(-) \circ_{L_{\bullet}} A: \mathcal{M}_{L_{\bullet}}^{I} \rightarrow \widetilde{\mathcal{M}}_{A}$ in the top row of (6.1) obeys the following properties.

(1) $\tilde{Y}$ reflects isomorphisms.

(2) Any $\widetilde{Y}$-contractible pair of morphisms (in the dual sense of 3, page 93, Section 3.3]) possesses a contractible (hence absolute) equalizer in $\mathcal{M}_{L_{\bullet}}^{I}$.

Proof. By a symmetric version of Lemma 3.5, $L_{\bullet}$ is a Frobenius monoid in $\mathcal{M}^{I}$. Applying the results about Frobenius monads in [27, Lemma 1.3 and Proposition 1.4], both of its category of modules, and its category of comodules are isomorphic to $\mathcal{M}_{L_{\bullet}}^{I}$; and the forgetful functor $V_{L_{\bullet}}: \mathcal{M}_{L_{\bullet}}^{I} \rightarrow \mathcal{M}^{I}$ possesses isomorphic left and right adjoints $(-) \circ L_{\bullet}$. Moreover, since $L_{\bullet}$ is a separable comonoid in $\mathcal{M}^{I}$, the forgetful functor $V_{L_{\bullet}}: \mathcal{M}_{L_{\bullet}}^{I} \rightarrow \mathcal{M}^{I}$ is separable by Rafael's theorem [24]: The unit of the adjunction $V_{L_{\bullet}} \dashv(-) \circ L_{\bullet}$ is given by the $L_{\bullet}$-coaction $\varrho: X \rightarrow X \circ L_{\bullet}$ at any object $X$ of $\mathcal{M}_{L_{\bullet}}^{I}$. It has a natural retraction given by the $L_{\bullet}$-action $X \circ L_{\bullet} \stackrel{\rho \circ L \bullet}{\longrightarrow} X \circ L_{\bullet} \circ L_{\bullet} \stackrel{X_{\bullet} \varepsilon_{\bullet}^{L} \mu_{\bullet}^{L}}{\longrightarrow} X$ on $X$.

The forgetful functor $U^{I}: \mathcal{M}^{I} \rightarrow \mathcal{M}$ has a right adjoint $(-) \bullet I: \mathcal{M} \rightarrow \mathcal{M}^{I}$ and $(-) \circ J: \mathcal{M} \rightarrow$ $\mathcal{M}_{J}$ is left adjoint of the forgetful functor $U_{J}: \mathcal{M}_{J} \rightarrow \mathcal{M}$. Thus the down-then-right path in (6.1) is a composite of left adjoint separable functors, hence it is a separable and left adjoint functor. 
Since idempotent morphisms are assumed to split in $\mathcal{M}$, they also split in $\mathcal{M}_{L_{\bullet}}^{I}$. Thus the claims follow by [17, Proposition 1.13]. (In fact, in [17, Proposition 1.13] part (2) of our proposition appears in a slightly different form. There the claim is stated about coreflexive $\tilde{Y}$-contractible equalizer pairs; though these additional assumptions are not used in their proof.)

With these preparations, the following 'fundamental theorem of Hopf modules' is obtained.

Theorem 6.2. Let $(\mathcal{M}, \circ, \bullet)$ be a duoidal category in which idempotent morphisms split and the functor $\mathcal{M}^{I} \stackrel{U^{I}}{\longrightarrow} \mathcal{M} \stackrel{(-) \circ J}{\longrightarrow} \mathcal{M}_{J}$ is separable. Let $A$ be a weak bimonoid in $\mathcal{M}$. The corresponding Galois morphism (5.9) is an isomorphism if and only if the functor $(-){ }^{\circ}{ }_{L_{\bullet}} A: \mathcal{M}_{L_{\bullet}}^{I} \rightarrow \mathcal{M}_{A}^{A}$ is an equivalence.

Proof. We use the reasoning applied in [17, Proposition 3.7] in the non-weak case, to show that $Y=(-) \circ_{L} A: \mathcal{M}_{L_{\bullet}}^{I} \rightarrow \mathcal{M}_{A}$ is comonadic.

Since $\tilde{Y}=(-) \circ_{L_{\bullet}} A: \mathcal{M}_{L_{\bullet}}^{I} \rightarrow \widetilde{\mathcal{M}}_{A}$ reflects isomorphisms by Proposition 6.1, clearly so does $Y=(-) \circ_{L} \cdot A: \mathcal{M}_{L_{\bullet}}^{I} \rightarrow \mathcal{M}_{A}$ differing from $\tilde{Y}$ by the fully faithful embedding $\widetilde{\mathcal{M}}_{A} \rightarrow \mathcal{M}_{A}$.

Since idempotent morphisms in $\mathcal{M}$ split, they also split in $\mathcal{M}_{A}$. So in this case a $Y$-contractible equalizer pair (in the dual sense of [3, page 94]) is simply a $Y$-contractible pair (in the dual sense of [3, page 93, Section 3.3]). Using again that $Y$ and $\widetilde{Y}$ differ by the fully faithful embedding $\widetilde{\mathcal{M}}_{A} \rightarrow$ $\mathcal{M}_{A}$, we conclude that a $Y$-contractible pair is the same as a $\widetilde{Y}$-contractible pair. By Proposition 6.1. $\widetilde{Y}$-contractible pairs (i.e. $Y$-contractible equalizer pairs) possess absolute equalizers. This proves that $Y$ creates equalizers of $Y$-contractible equalizer pairs.

Since there are adjunctions

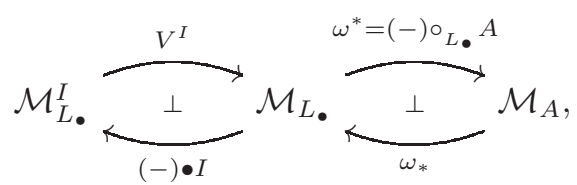

$Y$ (occurring in the top row) possesses a right adjoint. So it follows by the dual form of Beck's theorem [4] (see [3, page 100, Theorem 3.14]) that $Y$ is comonadic. Since $(-){ }^{\circ}{ }_{\bullet} A: \mathcal{M}_{L}^{I} \rightarrow \mathcal{M}_{A}^{A}$ is an equivalence if and only if (5.9) is a natural isomorphism and $Y$ is comonadic (see e.g. [15, Theorem 2.7] or [17, Theorem 1.10]), this completes the proof.

Applying all possible composites of the duality transformations $\bullet$, ○ and $*$ in Section 2, we obtain seven further symmetric variants of the equivalent conditions in Theorem 6.2. In a braided monoidal category — regarded as a duoidal category — whose idempotent morphisms split, half of them reduce to the usual definition of weak Hopf monoid in [22, 2]; and half of them reduce to the condition that the opposite weak bimonoid is a weak Hopf monoid. 
Proof of Lemma 2.4 (3).

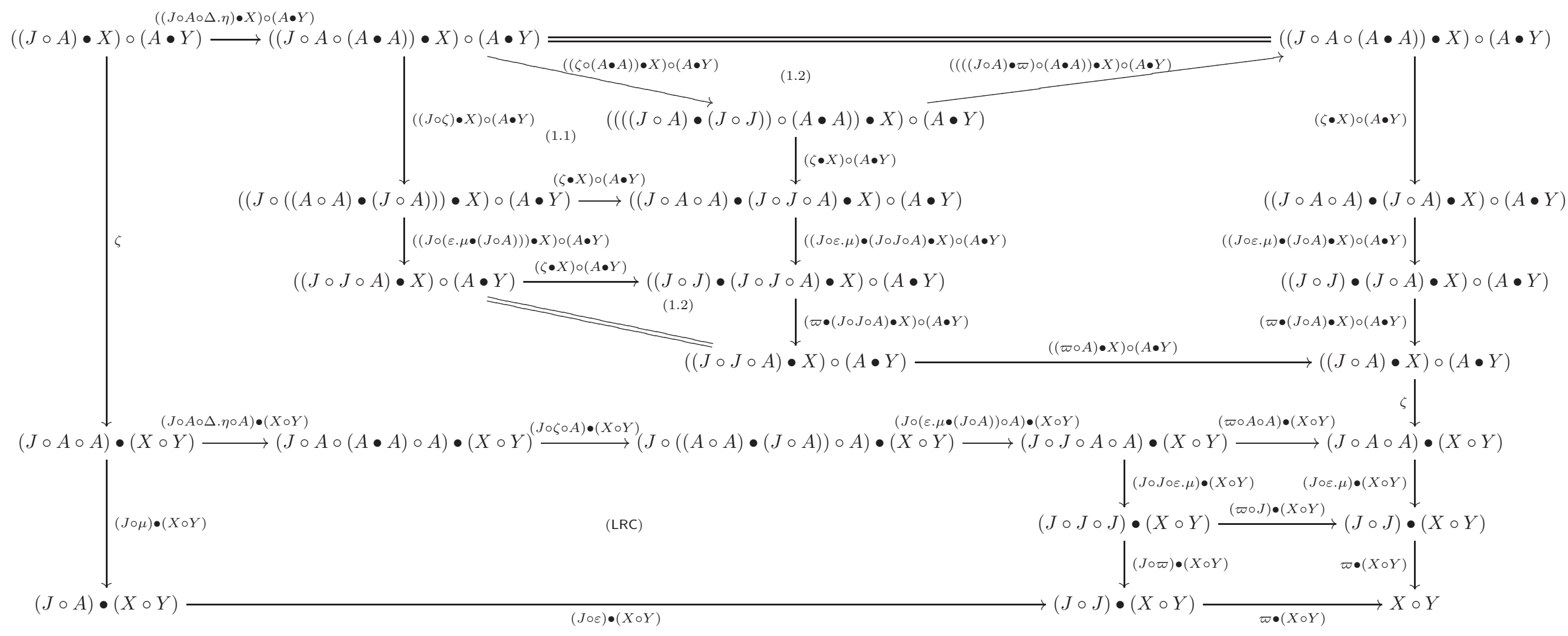


Proof of Theorem [2.5, verification of [8, eq. (1.5)].

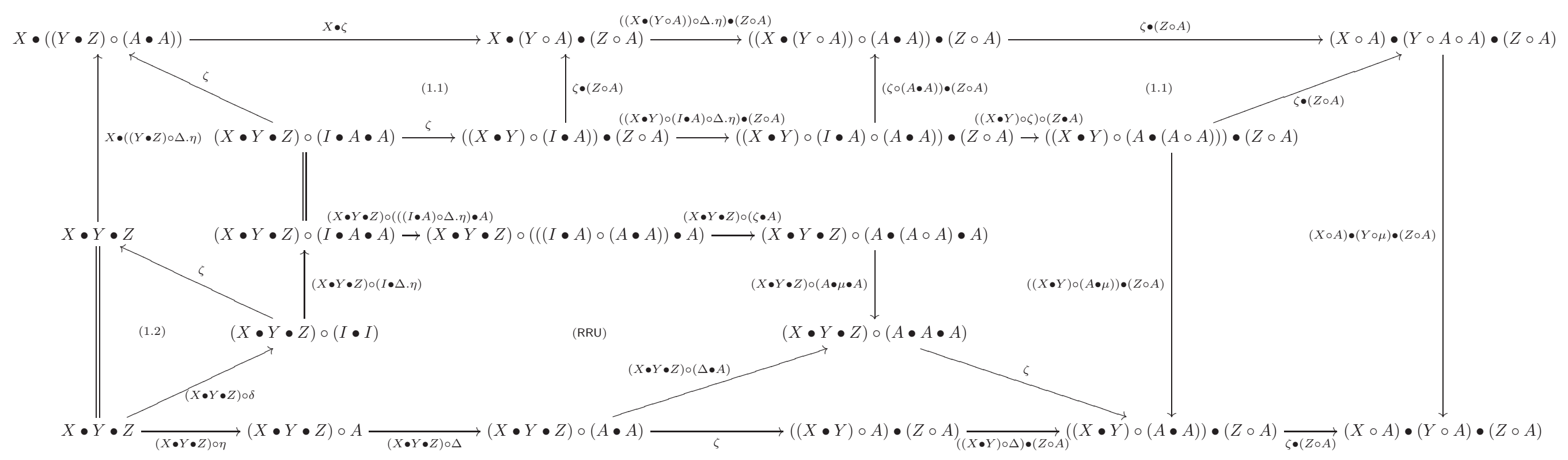


Proof of Theorem [2.5, verification of [8, eq. (1.4)].

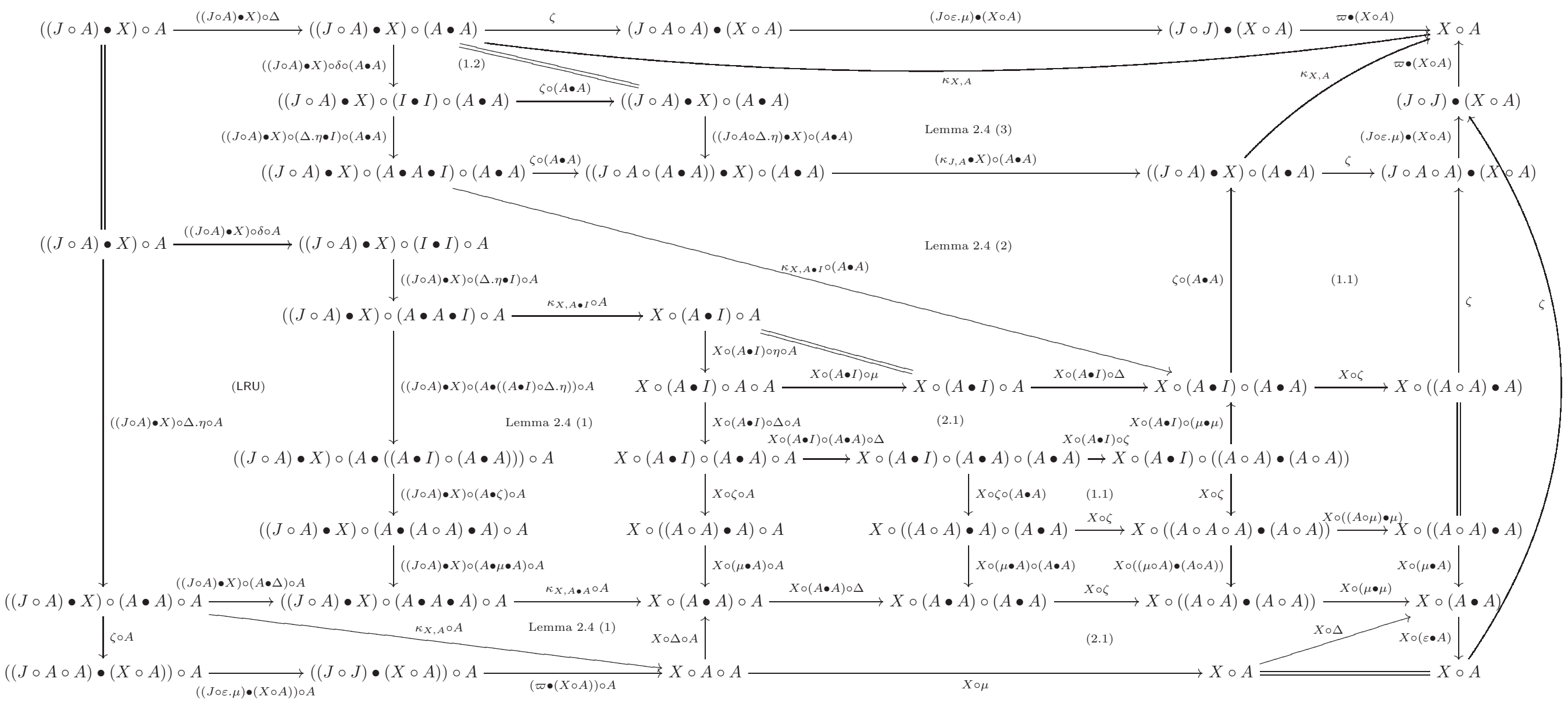


Proof of Lemma [3.1, computation of $\left((J \circ A) \bullet \sqcap_{\circ}^{R}\right) . \zeta .(J \circ \Delta) . \sqcap_{\circ}^{R}$.

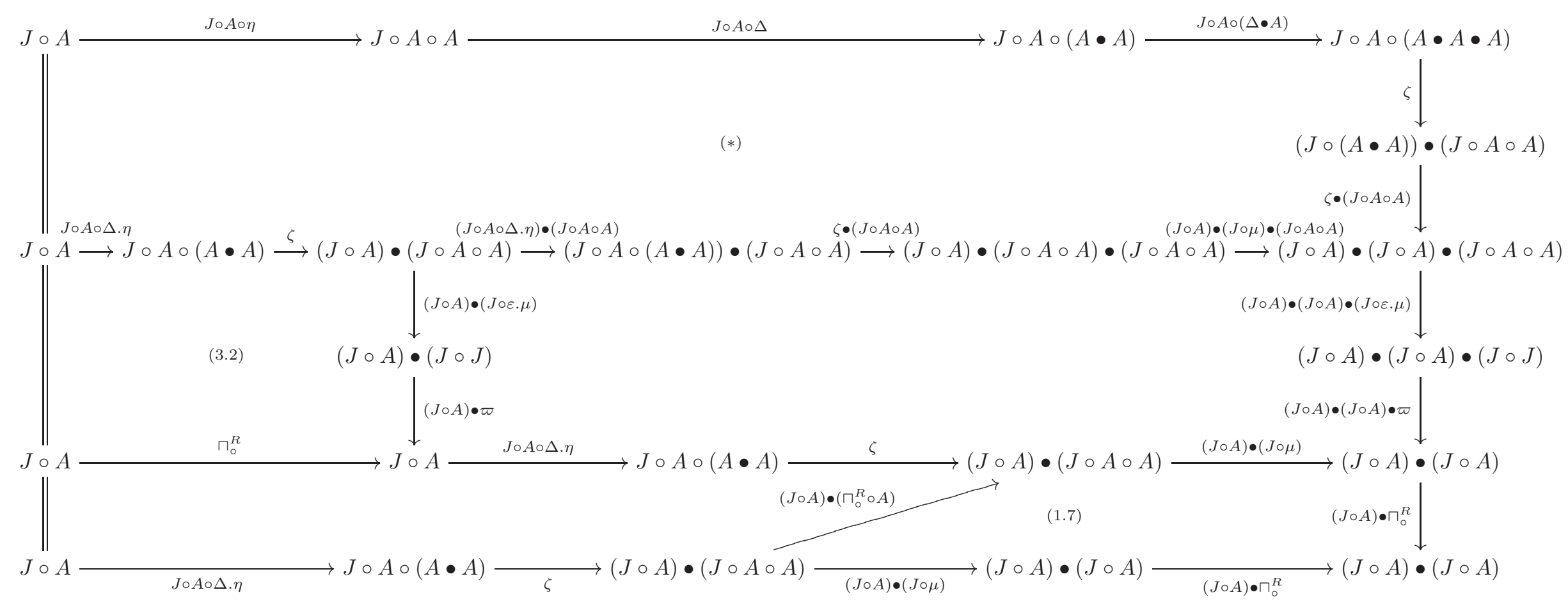


Proof of Lemma 3.1, computation of $\left(\Pi_{\circ}^{R} \bullet(J \circ A)\right) . \zeta .(J \circ \Delta)$.

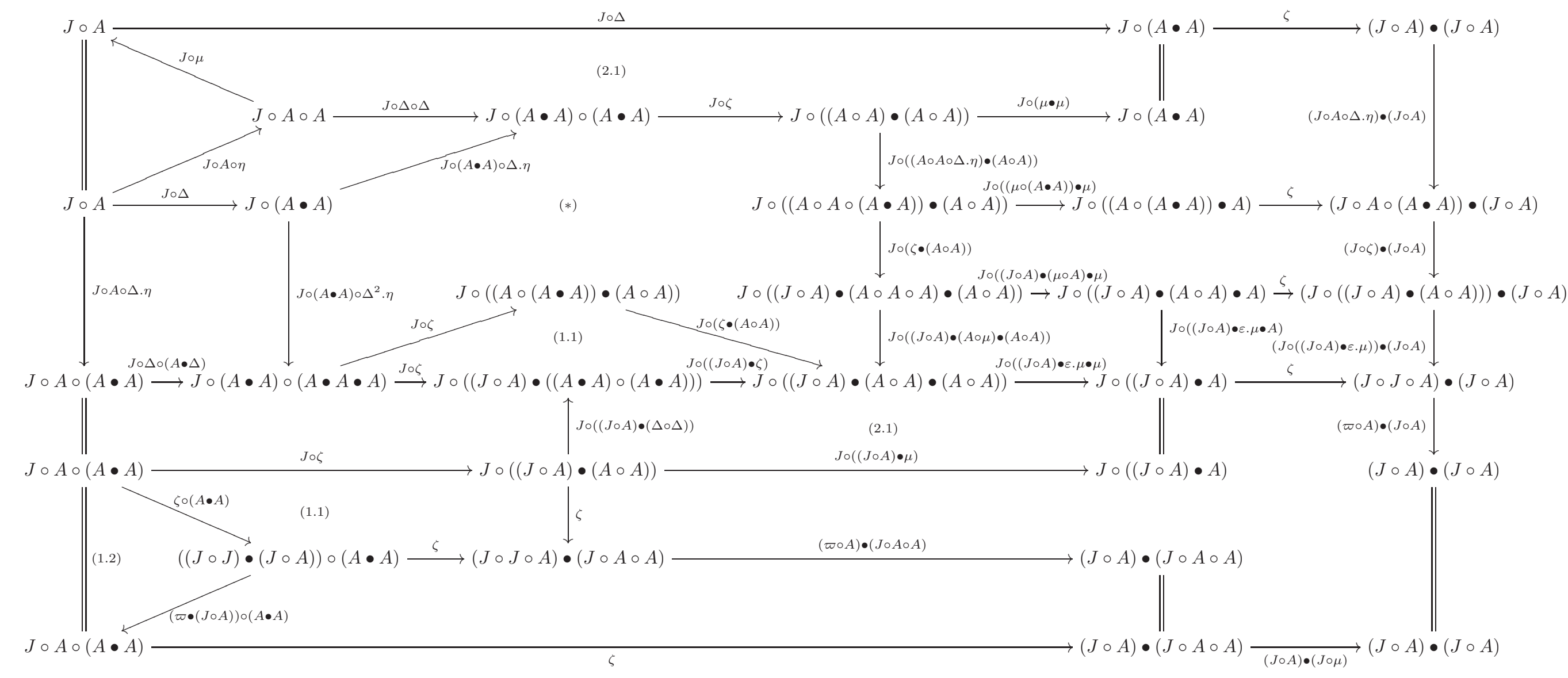




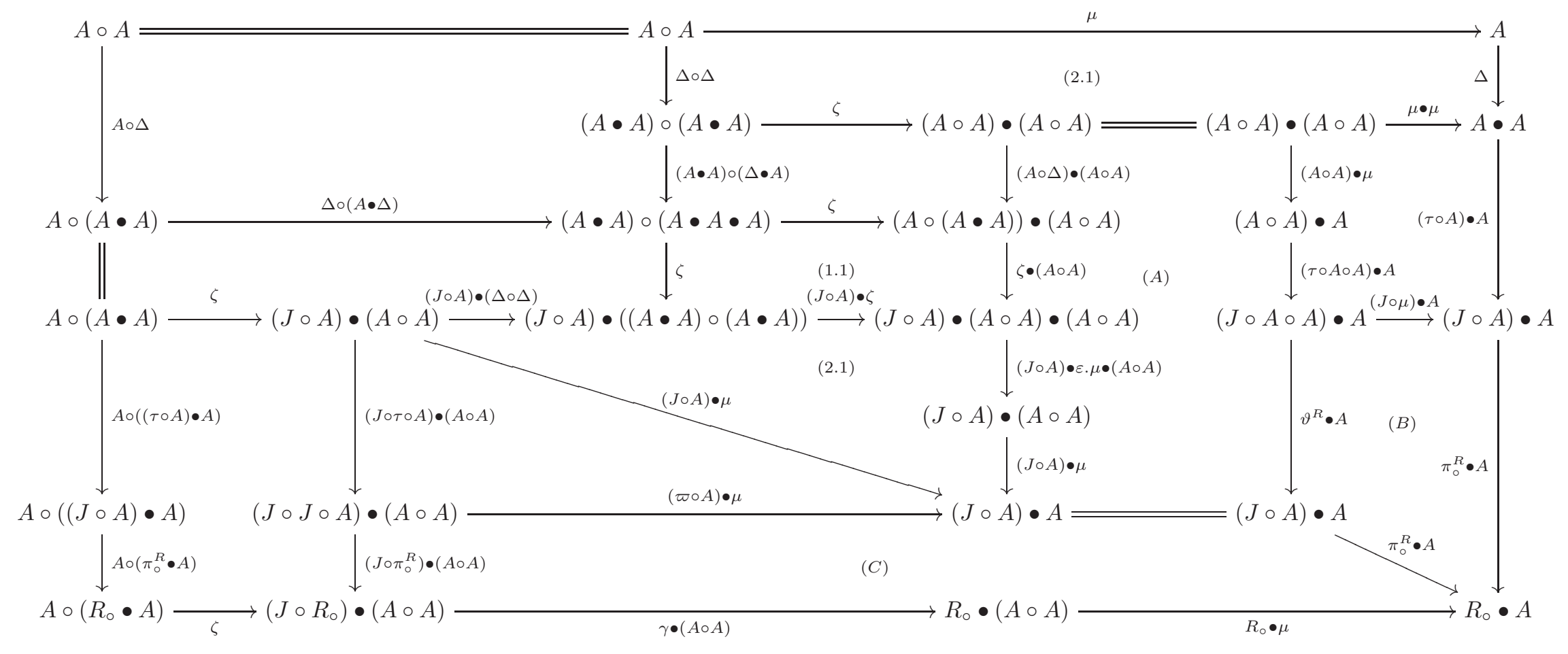


Proof of Proposition 5.1, verification of the compatibility of $\psi$ with the unit.

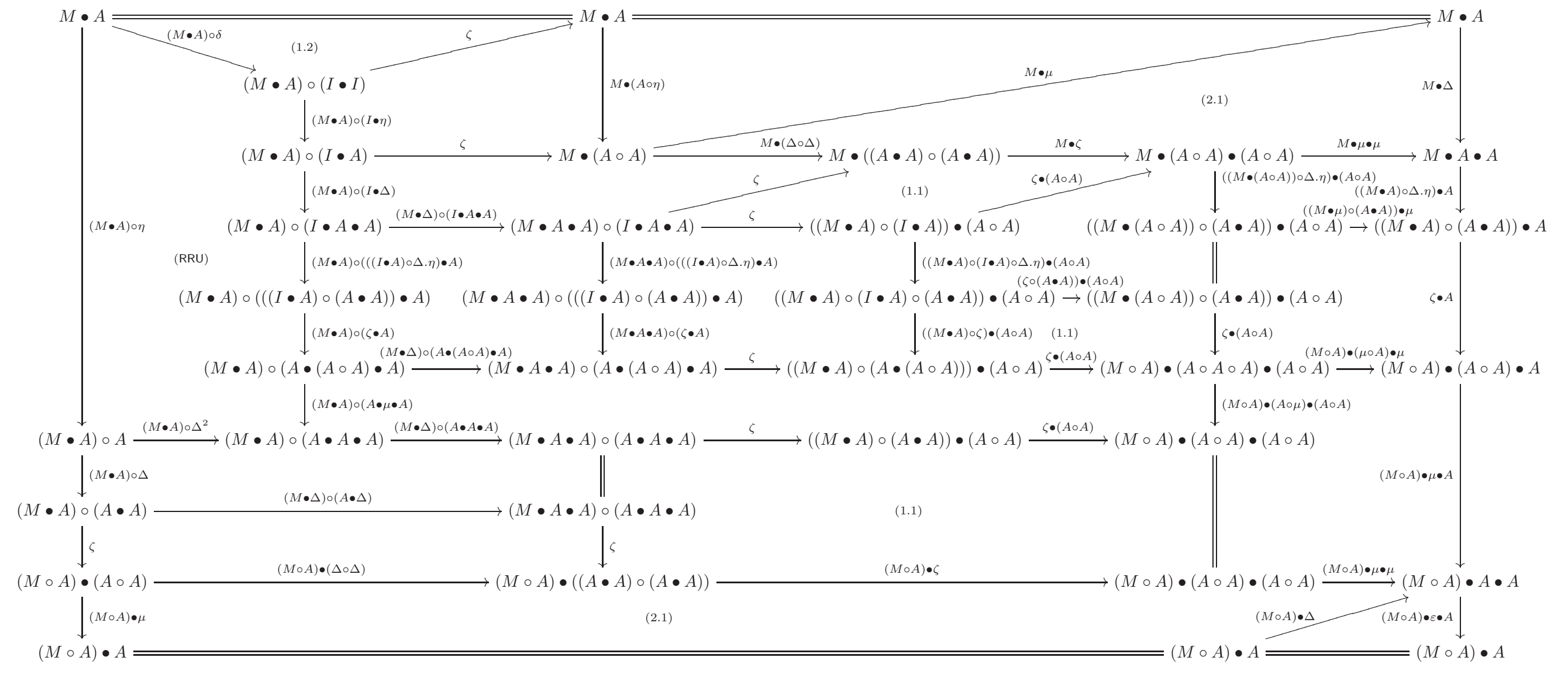


Proof of Lemma 5.4.

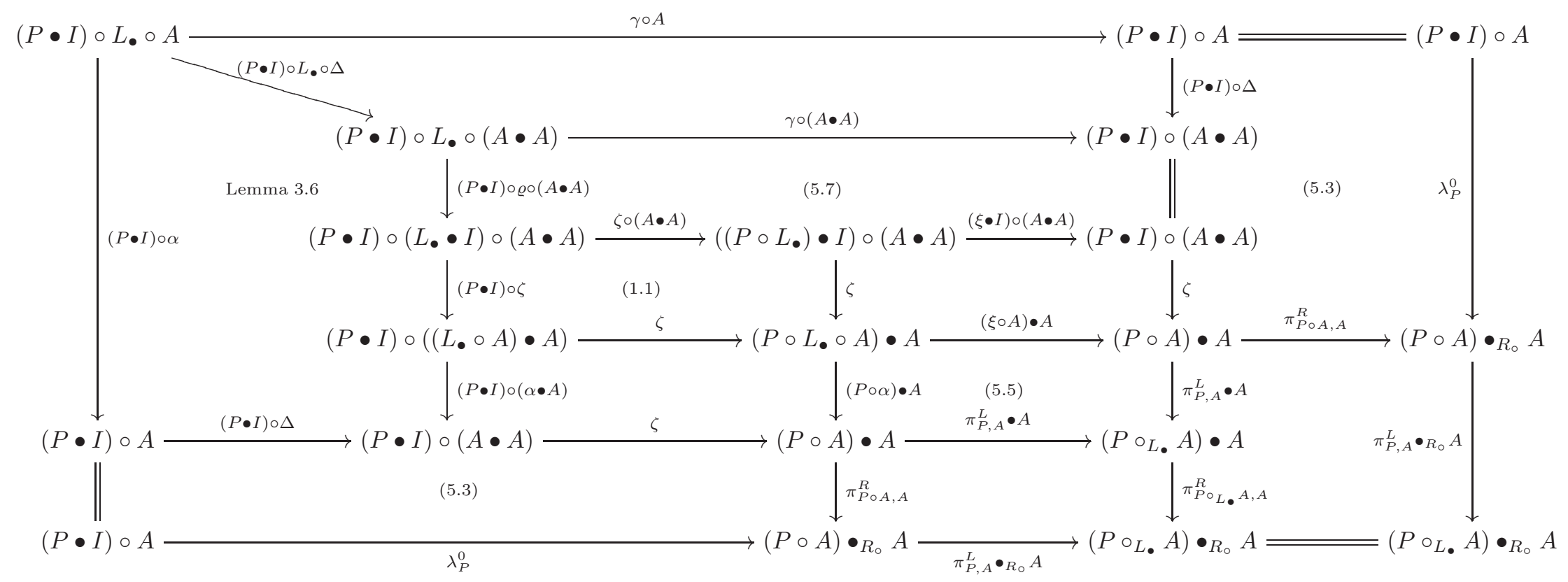




\section{REFERENCES}

[1] M. Aguiar and S. Mahajan, Monoidal Functors, Species and Hopf Algebras. CRM Monograph Series 29, American Math. Soc. Providence, 2010. Electronically available at: http://www.math.tamu.edu/ maguiar/a.pdf

[2] J.N. Alonso Álvarez, J.M. Fernández Vilaboa and R. González Rodríguez, Weak Hopf algebras and weak YangBaxter operators, J. Algebra 320, no. 5 (2008), 2101-2143.

[3] M. Barr and C. Wells, Toposes, Triples and Theories. Springer-Verlag, New York, (1985); available also in: Repr. Theory Appl. Categ. 12 (2005).

[4] J. Beck, Triples, Algebras and Cohomology. PhD Thesis, Columbia University (1967); available in: Repr. Theory Appl. Categ. 2 (2003).

[5] T. Booker and R. Street, Tannaka duality and convolution for duoidal categories, Theory Appl. Categ. 28, no. 6 (2013), 166-205.

[6] G. Böhm, The weak theory of monads, Adv. in Math. 225 (2010), 1-32.

[7] G. Böhm, Y.Y. Chen and L.Y. Zhang, On Hopf monoids in duoidal categories, preprint available at http://arxiv.org/abs/1212.1018v1

[8] G. Böhm, S. Lack and R. Street, Weak bimonads and weak Hopf monads, J. Algebra 328 (2011), 1-30.

[9] G. Böhm, S. Lack and R. Street, On the 2-categories of weak distributive laws, Comm. Algebra 39 no. 12 (2011), 4567-4583.

[10] G. Böhm, F. Nill and K. Szlachányi, Weak Hopf algebras. I. Integral theory and $C^{*}$-structure, J. Algebra 221 no. 2 (1999), 385-438.

[11] G. Böhm and K. Szlachányi, A coassociative $C^{*}$-quantum group with nonintegral dimensions, Lett. Math. Phys. 38 no. 4 (1996), 437-456.

[12] T. Brzeziński and S. Majid, Coalgebra bundles, Commun. Math. Phys. 191 no. 2 (1998), 467-492.

[13] T. Brzeziński and R. Wisbauer, Corings and comodules. Cambridge University Press 2003.

[14] S. Caenepeel and E. De Groot, Weak entwining structures and Doi-Hopf modules over weak Hopf algebras, [in:] "New Trends in Hopf Algebra Theory" N. Andruskiewitsch, W.R. Ferrer Santos and H.-J. Schneider (eds.) Contemp. Math. 267 pp. 31-54, American Math. Soc. Providence, 2000.

[15] J. Gómez-Torrecillas, Comonads and Galois Corings, Appl. Categor. Struct. 14 (2006), 579-598.

[16] M. McCurdy and R. Street, What separable Frobenius monoidal functors preserve?, Cah. Topol. Géom. Différ. Catég. 51 no. 1 (2010), 29-50.

[17] B. Mesablishvili and R. Wisbauer, Galois functors and generalised Hopf modules, preprint available at http://arxiv.org/abs/1302.1729v1

[18] I. Moerdijk, Monads on tensor categories, J. Pure Appl. Algebra 168 (2002), 189-208.

[19] C. Năstăsescu, M. Van den Bergh and F. Van Oystaeyen, Separable functors applied to graded rings, J. Algebra 123 (1989), 397-413.

[20] F. Nill, Weak bialgebras, preprint available at http://arxiv.org/abs/math/9805104

[21] B. Pareigis, Non-additive ring and module theory I. General theory of monoids. Publ. Math. Debrecen 24 (1977).

[22] C. Pastro and R. Street, Weak Hopf monoids in braided monoidal categories, Algebra Number Theory 3 no. 2 (2009), 149-207.

[23] J. Power and H. Watanabe, Combining a monad and a comonad, Theor. Computer Sci. 280 no. 1-2 (2002), $137-162$.

[24] M.D. Rafael, Separable functors revisited, Comm. Algebra 18 (1990), 1445-1459.

[25] P. Schauenburg, Weak Hopf algebras and quantum groupoids. [in:] "Noncommutative geometry and quantum groups" P.M. Hajac and W. Pusz (eds.) pp. 171-188, Banach Center Publ. 61 Polish Acad. Sci., Warsaw, 2003.

[26] R. Street, The formal theory of monads, J. Pure Appl. Algebra 2 (1972), 149-168.

[27] R. Street, Frobenius monads and pseudomonoids, J. Math. Phys. 45 no. 10 (2004), 3930-3948.

[28] K. Szlachányi, Adjointable monoidal functors and quantum groupoids, [in:] "Hopf algebras in noncommutative geometry and physics", S. Caenepeel and F. Van Oysaeyen (eds.), pp. 291-307, Lecture Notes in Pure and Appl. Math. 239, Dekker, New York, 2005. 
Department of Mathematics, Nanjing Agricultural University, Nanjing 210095, P.R. China. Wigner Research Centre for Physics, Budapest, H-1525 Budapest 114, P.O.B. 49, Hungary E-mail address: bohm.gabriella@wigner.mta.hu 